\title{
Stratifications with respect to actions of real reductive groups
}

\author{
Peter Heinzner, Gerald W. Schwarz and Henrik Stötzel
}

\begin{abstract}
We study the action of a real reductive group $G$ on a real submanifold $X$ of a Kähler manifold $Z$. We suppose that the action of $G$ extends holomorphically to an action of the complexified group $G^{\mathbb{C}}$ and that with respect to a compatible maximal compact subgroup $U$ of $G^{\mathbb{C}}$ the action on $Z$ is Hamiltonian. There is a corresponding gradient map $\mu_{\mathfrak{p}}: X \rightarrow$ $\mathfrak{p}^{*}$ where $\mathfrak{g}=\mathfrak{k} \oplus \mathfrak{p}$ is a Cartan decomposition of $\mathfrak{g}$. We obtain a Morse-like function $\eta_{\mathfrak{p}}:=\left\|\mu_{\mathfrak{p}}\right\|^{2}$ on $X$. Associated with critical points of $\eta_{\mathfrak{p}}$ are various sets of semistable points which we study in great detail. In particular, we have $G$-stable submanifolds $S_{\beta}$ of $X$ which are called pre-strata. In cases where $\mu_{\mathfrak{p}}$ is proper, the pre-strata form a decomposition of $X$ and in cases where $X$ is compact they are the strata of a Morse-type stratification of $X$. Our results are generalizations of results of Kirwan obtained in the case where $G=U^{\mathbb{C}}$ and $X=Z$ is compact.
\end{abstract}

\section{Introduction}

In this paper we continue our study of actions of real reductive groups on Kähler manifolds [HS07a, HS07b]. Roughly speaking, we extend Kirwan's results on actions of complex reductive groups [Kir84a] (see also [Nes84] for the projective case) to the real reductive case. We also obtain new results in the complex reductive case.

Let $G$ be a closed subgroup of the complex reductive group $H$. We say that $G$ is real reductive if there is a maximal compact subgroup $U$ of $H$ such that $K \times \mathfrak{p} \rightarrow G,(k, \beta) \mapsto k \exp \beta$, is a diffeomorphism. Here $K:=G \cap U$ is a maximal compact subgroup of $G$ and $\mathfrak{p}:=\mathfrak{g} \cap$ iu where $\mathfrak{u}$ denotes the Lie algebra of $U$ and $\mathfrak{g}$ the Lie algebra of $G$. The Lie algebra $\mathfrak{h}$ of $H$ is the direct sum $\mathfrak{u} \oplus \mathfrak{i} \mathfrak{u}$. We also say that $G$ is compatible with the Cartan decomposition $H=U^{\mathbb{C}}=U$ exp iu of $H$. In this paper we fix $U$ and the real reductive subgroup $G \subset H$.

Assume that $H$ acts holomorphically on a complex Kähler manifold $Z$, that the Kähler form $\omega$ is $U$-invariant and that there is a $U$-equivariant moment mapping $\mu: Z \rightarrow \mathfrak{u}^{*}$. For $\xi \in \mathfrak{u}$ and $z \in Z$ we set $\mu^{\xi}(z):=\langle\mu(z), \xi\rangle:=\mu(z)(\xi)$. The inclusion ip $\hookrightarrow \mathfrak{u}$ induces by restriction a $K$-equivariant map $\mu_{\text {ip }}: Z \rightarrow(\mathfrak{i p})^{*}$. Using a $U$-invariant inner product on $\mathfrak{h}$ we can identify (ip $)^{*}$ and $\mathfrak{p}$, so we view $\mu_{\text {ip }}$ as a map $\mu_{\mathfrak{p}}: Z \rightarrow \mathfrak{p}$. For $\beta \in \mathfrak{p}$ let $\mu_{\mathfrak{p}}^{\beta}$ denote $\mu^{-\mathrm{i} \beta}$. Then $\operatorname{grad} \mu_{\mathfrak{p}}^{\beta}=\beta_{Z}$ where $\beta_{Z}$ is the vector field on $Z$ corresponding to $\beta$ and grad is computed with respect to the Riemannian metric induced by the Kähler structure. We call $\mu_{\mathfrak{p}}$ the G-gradient map associated with $\mu$.

Received 23 January 2007, accepted in final form 16 July 2007, published online 23 January 2008.

2000 Mathematics Subject Classification 32M05.

Keywords: Cartan decomposition, Hamiltonian action, moment map, stratification.

The first author is partially supported by the Sonderforschungsbereich SFB/TR12 of the Deutsche Forschungsgemeinschaft. The second author is partially supported by NSA Grant H98230-06-1-0023. The third author is supported by the Sonderforschungsbereich SFB/TR12 of the Deutsche Forschungsgemeinschaft.

This journal is (C) Foundation Compositio Mathematica 2008. 


\section{P. Heinzner, G. W. Schwarz and H. Stötzel}

For the rest of this paper we fix a $G$-stable locally closed real submanifold $X$ of $Z$. We may consider $\mu_{\mathfrak{p}}$ as a mapping $\mu_{\mathfrak{p}}: X \rightarrow \mathfrak{p}$ such that

$$
\operatorname{grad} \mu_{\mathfrak{p}}^{\beta}=\beta_{X}
$$

where the gradient is now computed with respect to the induced Riemannian metric on $X$. Since $X$ is $G$-stable we have $\beta_{Z}(z)=\beta_{X}(z)$ for $z \in X$. Using the inner product on $\mathfrak{p} \subset$ iu we define $\eta_{\mathfrak{p}}(z):=\frac{1}{2}\left\|\mu_{\mathfrak{p}}(z)\right\|^{2}, z \in X$. Let $\mathcal{C}_{\mathfrak{p}}$ be the set of critical points of $\eta_{\mathfrak{p}}$ and $\mathcal{B}_{\mathfrak{p}}:=\mu_{\mathfrak{p}}\left(\mathcal{C}_{\mathfrak{p}}\right) \subset \mathfrak{p}$.

A strategy for analyzing the $G$-action on $X$ is to view $\eta_{\mathfrak{p}}$ as a generalized Morse function in order to obtain a smooth $G$-stable stratification of $X$ and to study its properties, as follows. Let $\varphi_{t}$ denote the flow of the vector field $\operatorname{grad} \eta_{\mathfrak{p}}$. For $\beta \in \mathcal{B}_{\mathfrak{p}}$ consider the set $S_{\beta}^{\prime}:=\left\{x \in X \mid \varphi_{t}(x)\right.$ has a limit point in $\mathcal{C}_{\mathfrak{p}} \cap \mu_{\mathfrak{p}}^{-1}(K \cdot \beta)$ as $t$ goes to $\left.-\infty\right\}$. From the Morse theoretical point of view, the set $S_{\beta}^{\prime}$ is a candidate for a Morse-stratum associated with $\eta_{\mathfrak{p}}$. Difficulties arise, since $\mathcal{C}_{\mathfrak{p}}$ is almost never smooth, the flow $\varphi_{t}$ may not exist for all $t \leqslant 0$ and if it exists a limit point might not be unique.

We get around these difficulties by defining the relevant sets, which we call pre-strata, in terms of semistability. Set $\mathcal{M}_{\mathfrak{p}}:=\mu_{\mathfrak{p}}^{-1}(0)$ and $\mathcal{S}_{G}\left(\mathcal{M}_{\mathfrak{p}}\right):=\left\{z \in X: \overline{G \cdot z} \cap \mu_{\mathfrak{p}}^{-1}(0) \neq \emptyset\right\}$, the (open) set of semistable points in $X$. If $\beta=0 \in \mathcal{B}_{\mathfrak{p}}$, then we define $S_{\beta}=S_{0}=\mathcal{S}_{G}\left(\mathcal{M}_{\mathfrak{p}}\right)$. We define $S_{\beta}$ for any $\beta \in \mathcal{B}_{\mathfrak{p}}$ in a similar way, as follows.

For $\beta \in \mathfrak{p}$ let $G^{\beta_{+}}:=\left\{g \in G: \lim _{t \rightarrow-\infty} \exp (t \beta) g \exp (-t \beta)\right.$ exists $\}$. Then $G^{\beta_{+}}$is parabolic with Levi component the centralizer $G^{\beta}$ of $\beta$. Note that $G^{\beta}=K^{\beta} \exp \mathfrak{p}^{\beta}$ is compatible with the Cartan decomposition of $U^{\mathbb{C}}$. Let $X^{\beta}$ denote the set of zeros of the vector field $\beta_{X}$. This submanifold is stable with respect to the action of $G^{\beta}$ and we have a shifted gradient map $\widehat{\mu_{\mathfrak{p}^{\beta}}}: X^{\beta} \rightarrow \mathfrak{p}^{\beta}$, $x \mapsto \mu_{\mathfrak{p}^{\beta}}(x)-\beta$, whose set of semistable points is $\widehat{S_{0}}:=\left\{x \in X^{\beta}: \overline{G^{\beta} \cdot x} \cap \mu_{\mathfrak{p}^{\beta}}^{-1}(\beta) \neq \emptyset\right\}$. Set $S^{\beta_{+}}:=\left\{z \in X: \lim _{t \rightarrow-\infty} \exp (t \beta) \cdot z \in \widehat{S_{0}}\right\}$. Then $S^{\beta_{+}}$is $G^{\beta_{+}}$-stable. We call $S_{\beta}:=G \cdot S^{\beta_{+}}$the pre-stratum associated with $\beta \in \mathcal{B}_{\mathfrak{p}}$.

Let $G \times G^{\beta_{+}} S^{\beta_{+}}$denote the quotient of $G \times S^{\beta_{+}}$with respect to the $G^{\beta_{+}}$-action which is given by $q \cdot(g, s)=\left(g q^{-1}, q \cdot s\right)$ for $g \in G, s \in S^{\beta_{+}}, q \in G^{\beta_{+}}$. In $\S 5$ we obtain our main results concerning pre-strata.

Slice Theorem. The pre-stratum $S_{\beta}$ associated with $\beta$ is a locally closed submanifold of $X$ and the natural map

$$
G \times{ }^{G^{\beta}} S^{\beta_{+}} \rightarrow S_{\beta}
$$

induced by the $G$-action on $X$ is a diffeomorphism.

The $G$-action on a pre-stratum has properties similar to those of $G$-actions on a set of semistable points. The role of the zero fiber of the gradient map is taken over by the $\operatorname{set} \mathcal{M}_{\mathfrak{p}}(K \cdot \beta):=\mu_{\mathfrak{p}}^{-1}(K \cdot \beta)$. Our second result (Theorem 5.4 and the subsequent discussion) is the following.

Quotient Theorem. Let $\beta \in \mathcal{B}_{\mathfrak{p}}$.

(i) The topological Hilbert quotients (see $\S 3$ ) $S^{\beta_{+}} / / G^{\beta}$ and $S_{\beta} / / G$ exist and are isomorphic and parameterize the closed orbits.

(ii) The inclusions $\mathcal{M}_{\mathfrak{p}^{\beta}}(\beta) \cap S^{\beta_{+}} \subset S^{\beta_{+}}$and $\mathcal{M}_{\mathfrak{p}}(K \cdot \beta) \cap S_{\beta} \subset S_{\beta}$ induce isomorphisms $\left(\mathcal{M}_{\mathfrak{p}^{\beta}}(\beta) \cap\right.$ $\left.S^{\beta_{+}}\right) / K^{\beta} \cong S^{\beta_{+}} / / G^{\beta}$ and $\left(\mathcal{M}_{\mathfrak{p}}(K \cdot \beta) \cap S_{\beta}\right) / K \cong S_{\beta} / / G$.

(iii) The set of minima of $\eta_{\mathfrak{p}} \mid S_{\beta}$ coincides with $\mathcal{M}_{\mathfrak{p}}(K \cdot \beta) \cap S_{\beta}$. 


\section{STRATIFICATIONS}

The content of these results can be summarized as in the following commutative diagram.

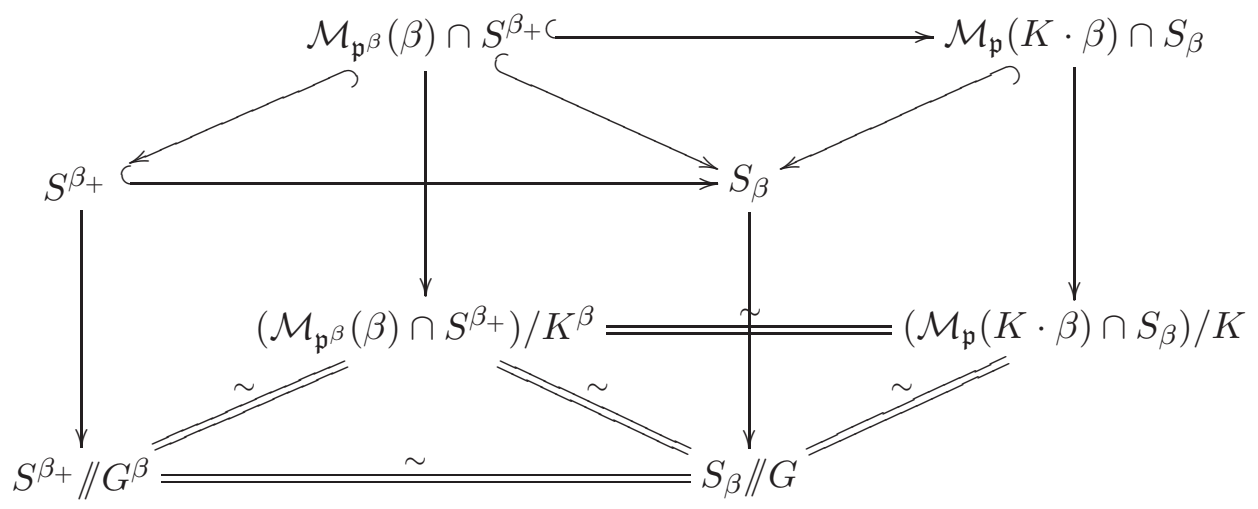

We also show in $\S 5$ that a pre-stratum $S_{\beta}$ only depends upon $K \cdot \beta$.

Stratification Theorem (Theorem 5.5). Let $\beta, \tilde{\beta} \in \mathcal{B}_{\mathfrak{p}}$.

(i) If $S_{\tilde{\beta}} \cap \overline{S_{\beta}} \neq \emptyset$ and $S_{\beta} \neq S_{\tilde{\beta}}$, then $\|\tilde{\beta}\|>\|\beta\|$.

(ii) The following are equivalent:

(a) $K \cdot \beta=K \cdot \tilde{\beta}$;

(b) $S_{\beta}=S_{\tilde{\beta}}$;

(c) $S_{\beta} \cap S_{\tilde{\beta}} \neq \emptyset$.

If $\mu_{\mathfrak{p}}: X \rightarrow \mathfrak{p}$ is a proper map, then we show in $\S 7$ that for $\beta \in \mathcal{B}_{\mathfrak{p}}$, the Morse-theoretical stratum $S_{\beta}^{\prime}$ coincides with the pre-stratum $S_{\beta}$. Moreover, we prove the following.

Theorem (Theorem 7.3). If $\mu_{\mathfrak{p}}: X \rightarrow \mathfrak{p}$ is proper, then $X=\bigcup_{\beta} S_{\beta}$ where $\beta$ runs through a complete set of representatives of $K$-orbits in $\mathcal{B}_{\mathfrak{p}}$.

In $\S 8$ we obtain the following.

Theorem (Corollary 8.5). If $X$ is compact, then there are only finitely many pre-strata.

If $X=Z$ is compact and $G=U^{\mathbb{C}}$, then most of the above is proved in [Kir84a]. This holomorphic and compact case has been studied quite intensively. For example, the strata $S_{\beta}$ are smooth analytic subsets of $Z$ which are open in their closure with respect to the analytic Zariski topology, there is only one open pre-stratum $S_{\beta_{0}}$ (if $X$ is connected) and its complement is a closed analytic subset of $X$. Also, the open pre-stratum $S_{\beta_{0}}$ is connected and is minimal in the sense that $\beta_{0}$ is a closest point to zero in $\mu_{\mathfrak{p}}(X) \subset \mathfrak{p}$. Many of these results about open pre-strata hold more generally for meromorphic actions of complex reductive groups on compact Kähler manifolds (see [Fuj78, Som75]).

Important applications noted in [Kir84a] are the Morse equalities in equivariant cohomology in the holomorphic compact case. Morse inequalities also hold in our setup for general real reductive group actions on compact manifolds. We illustrate in $\S 9$ with simple examples that equalities are almost never valid for actions of real reductive groups.

The simplest interesting situation where our results apply and that is not covered by previously known results is the real projective case. Here $X \subset \mathbb{P}\left(V_{\mathbb{R}}\right)$ where $V_{\mathbb{R}}$ is a representation space of an algebraic semisimple real group $G$ and $X$ is $G$-stable and closed. In this setup we may choose $Z=\mathbb{P}(V)$ where $V=V_{\mathbb{R}} \otimes \mathbb{C}$ and $\mathbb{P}(V)$ is given the Fubini-Study metric.

The special case $X=\mathbb{P}\left(V_{\mathbb{R}}\right)$ and $G$ real semisimple is treated in [Mar01]. However, there is an error in the proof of Marian's Theorem 1: the assumption on the bottom of p. 783 of [Mar01] that one may assume that $k_{1}=k_{2}=e$ is incorrect. 


\section{P. Heinzner, G. W. Schwarz and H. Stötzel}

Still in the projective setup we have the following result of Schützdeller [Sch06] which is new even in the case that $G=U^{\mathbb{C}}$ and which generalizes results of Kostant [Kos73], Atiyah [Ati82], Guillemin and Sternberg [GS82], Mumford (see the Appendix of [Nes84]), Kirwan [Kir84b] and O'Shea and Sjamaar [OSO0].

Let $\mathfrak{a}_{+}$be a positive Weyl chamber for the $K$-action on $\mathfrak{p}$. Let $Y$ be a semi-algebraic closed $G$-stable subset of $\mathbb{P}(V)$ which is irreducible (in a suitable sense). Then $\mu_{\mathfrak{p}}(Y) \cap \mathfrak{a}_{+}$is a convex polytope.

The proof of this convexity result makes essential use of the stratification obtained here.

Another related interesting simple case is where $X=Z$ is $U$-homogeneous and $G$ is a real form of $U^{\mathbb{C}}$. Our results applied to $G$ and also to the complex reductive group $K^{\mathbb{C}}$ imply Matsuki duality [BL02, Mat82, MUV92] (see Corollary 6.14) and may be viewed as a generalization thereof.

Most of our results in the holomorphic non-compact case are new. In this setup $G$ is a complex reductive group and $X$ is a Kähler manifold. The relevant maps are holomorphic and the quotients discussed above are Kähler spaces. In particular, a pre-stratum $S_{\beta}$ is $G$-equivariantly and biholomorphically identified with a holomorphic $G$-bundle over the compact complex manifold $G / G^{\beta_{+}}$.

Also, in the general real case, a pre-stratum $S_{\beta}$ fibers over the $G$-homogeneous compact manifold $G / G^{\beta_{+}}$which now is a $K$-homogeneous Riemannian manifold. The open pre-strata are of special interest. In many situations, including the case where $\mu_{\mathfrak{p}}$ has closed image, there is a minimal prestratum $S_{\beta_{0}}$ in the sense that $\beta_{0}$ is a closest point to zero in $\mu_{\mathfrak{p}}(X)$. This pre-stratum is always open and usually serves as a good substitute for the set of semistable points which quite often is empty.

In principle there could be many different minimal pre-strata and also other open pre-strata which are not minimal in the above sense. We do not know any example of a connected manifold $X$ where this is indeed the case. Any such example would imply that $\mu_{\mathfrak{p}}(X) \cap \mathfrak{a}_{+}$is not a convex subset of $\mathfrak{a}_{+}$. In situations where the convexity result of Schützdeller applies, the minimal pre-stratum is unique. The uniqueness of the minimal pre-stratum holds in complete generality if $X=Z$ and $G=U^{\mathbb{C}}$. In this case very general convexity theorems are available [HH96].

Another interesting extreme situation occurs when there is a maximal pre-stratum $S_{\beta}$. By this we mean that $\beta \in \mu_{\mathfrak{p}}(X)$ and $\|\beta\| \geqslant\left\|\mu_{\mathfrak{p}}(z)\right\|$ for all $z \in X$. In this case the function $\eta_{\mathfrak{p}}$ is constant on $S_{\beta}$ and we explain in $\S 6$ that the general theory implies that $S_{\beta}=\mathcal{M}_{\mathfrak{p}}(K \cdot \beta)=\mu_{\mathfrak{p}}^{-1}(K \cdot \beta)$ and that $S^{\beta_{+}}=\mathcal{M}_{\mathfrak{p}}(\beta)=\mu_{\mathfrak{p}}^{-1}(\beta)$. Every $G$-orbit in $S_{\beta}$ is a $K$-orbit and every $G^{\beta}$-orbit in $S^{\beta_{+}}$is a $K^{\beta}$-orbit. Moreover, $S^{\beta_{+}}=\mathcal{M}_{\mathfrak{p}}(\beta)$ is a union of connected components of $X^{\beta}$ and the Slice Theorem gives a $K$-equivariant diffeomorphism $K \times{ }^{K^{\beta}} \mathcal{M}_{\mathfrak{p}}(\beta) \cong S_{\beta}$. These considerations generalize an old theorem of Wolf [Wol69] (see Corollary 6.16) which says that a real form $G$ of a complex semisimple group acting on a generalized flag manifold has a unique closed $G$-obit.

\section{Compatible subgroups and gradient maps}

Let $U$ be a compact Lie group. Then $U$ has a natural real linear algebraic group structure, and we denote by $U^{\mathbb{C}}$ the corresponding complex linear algebraic group [Che46]. The group $U^{\mathbb{C}}$ is reductive and is the universal complexification of $U$ in the sense of [Hoc65]. On the Lie algebra level we have the Cartan decomposition $\mathfrak{u}^{\mathbb{C}}=\mathfrak{u}+\mathrm{iu}$ with a corresponding Cartan involution $\theta: \mathfrak{u}^{\mathbb{C}} \rightarrow \mathfrak{u}^{\mathbb{C}}$, $\xi+\mathrm{i} \eta \mapsto \xi-\mathrm{i} \eta, \xi, \eta \in \mathfrak{u}$. The real analytic map $U \times \mathrm{i} \mathfrak{u} \rightarrow U^{\mathbb{C}},(u, \xi) \mapsto u \exp \xi$, is a diffeomorphism. We refer to the decomposition $U^{\mathbb{C}}=U \exp (\mathrm{iu}) \cong U \times \mathfrak{i} \mathfrak{u}$ as the Cartan decomposition of $U^{\mathbb{C}}$, and we fix it for the remainder of this paper.

Let $G$ be a real Lie subgroup of $U^{\mathbb{C}}$. We say that $G$ is a compatible subgroup of $U^{\mathbb{C}}$ if it is compatible with the Cartan decomposition of $U^{\mathbb{C}}$, i.e. if

$$
K \times \mathfrak{p} \rightarrow G, \quad(k, \beta) \mapsto k \exp \beta,
$$




\section{StRATificATIONS}

is a diffeomorphism where $K=G \cap U$ and $\mathfrak{p}=\mathfrak{g} \cap \mathfrak{i} \mathfrak{u}$. In particular, $G$ is a closed subgroup of $U^{\mathbb{C}}$ if and only if $K$ is compact.

Let $Z$ be a smooth holomorphic $U^{\mathbb{C}}$-space, i.e. $Z$ is a complex manifold with a holomorphic action $U^{\mathbb{C}} \times Z \rightarrow Z$. We assume that $Z$ has a Kähler form $\omega$ which is $U$-invariant. We also assume that we are given a $U$-equivariant moment mapping $\mu: Z \rightarrow \mathfrak{u}^{*}$. By definition we then have

$$
d \mu^{\xi}=\imath_{\xi_{Z}} \omega
$$

where $\mu^{\xi}$ is $\mu$ followed by evaluation at $\xi \in \mathfrak{u}$ and $\xi_{Z}$ is the vector field on $Z$ with one parameter group $(t, z) \mapsto \exp t \xi \cdot z$.

Now let $G$ be a compatible closed subgroup of $U^{\mathbb{C}}$. We have the subspace ip $\subset \mathfrak{u}$ and a corresponding mapping $\mu_{\mathrm{ip}}: Z \rightarrow(\mathrm{ip})^{*}$ where $\mu_{\mathrm{ip}}$ is just $\mu$ followed by restriction to ip. The map $\mu_{\mathrm{ip}}$ is the correct analogue of $\mu$ when one is considering the action of $G$ rather than the action of $U^{\mathbb{C}}$. In order to simplify notation we replace consideration of $\mu_{\mathrm{ip}}$ by that of $\mu_{\mathfrak{p}}: Z \rightarrow \mathfrak{p}$ where

$$
\mu_{\mathfrak{p}}^{\beta}(z):=\left\langle\mu_{\mathfrak{p}}(z), \beta\right\rangle:=\mu_{\mathrm{ip}}^{-\mathrm{i} \beta}(z) .
$$

Here $\langle\cdot, \cdot\rangle$ denotes a $K$-invariant inner product on $\mathfrak{p} \subset \mathrm{i}$. The map $\mu_{\mathfrak{p}}$ is called the $G$-gradient map associated with $\mu$ since the equation $d \mu^{\xi}=\imath_{\xi_{Z}} \omega$ is equivalent to $\operatorname{grad} \mu_{\mathfrak{p}}^{\beta}=\beta_{Z}$ for all $\beta \in \mathfrak{p}$. Here the gradient is computed with respect to the Riemannian metric $(\cdot, \cdot)$ on $Z$ given by $(v, w)=\omega(v, J w)$ for all $z \in Z$ and $v, w \in T_{z}(Z)$ where $J$ denotes the complex structure on $T(Z)$.

For the rest of this paper we fix a $G$-stable locally closed submanifold $X$ of $Z$. Of course, a special important case is where $X=Z$. From now on we also denote the restriction of $\mu_{\mathfrak{p}}$ to $X$ by $\mu_{\mathfrak{p}}$. We have

$$
\operatorname{grad} \mu_{\mathfrak{p}}^{\beta}=\beta_{X}
$$

where grad is computed with respect to the induced Riemannian structure. Similarly, $\perp$ denotes perpendicularity relative to the induced Riemannian metric on $X$. We have the following two elementary results (see [HS07a, Lemmas 5.1 and 5.4]).

Lemma 2.1. Let $x \in X$ and $\beta \in \mathfrak{p}$. Then either $\beta_{X}(x)=0$ or the function $t \rightarrow \mu_{\mathfrak{p}}^{\beta}(\exp t \beta \cdot x)$ is strictly increasing.

For a subspace $\mathfrak{m}$ of $\mathfrak{u}^{\mathbb{C}}$ and $x \in X$ let $\mathfrak{m} \cdot x$ denote the subspace $\left\{\beta_{X}(x): \beta \in \mathfrak{m}\right\}$ of the tangent space $T_{x}(X)$.

Lemma 2.2. For all $x \in X$ we have $\operatorname{ker} d \mu_{\mathfrak{p}}(x)=(\mathfrak{p} \cdot x)^{\perp}$.

We use $\|\cdot\|$ to denote the norm functions associated to $\langle\cdot, \cdot\rangle$ and $(\cdot, \cdot)$. The critical points of the norm square function $\eta_{\mathfrak{p}}(x):=\frac{1}{2}\left\|\mu_{\mathfrak{p}}(x)\right\|^{2}$ will be of central importance in the rest of this paper.

Lemma 2.3. Let $x \in X$ and $\beta=\mu_{\mathfrak{p}}(x)$. Then $\operatorname{grad} \eta_{\mathfrak{p}}(x)=\beta_{X}(x)$.

Proof. This follows from $d \eta_{\mathfrak{p}}(x)(v)=\left\langle\beta, d \mu_{\mathfrak{p}}(x) v\right\rangle=d \mu_{\mathfrak{p}}^{\beta}(x)(v)=\left(v, \beta_{X}(x)\right)$.

Corollary 2.4. Let $x \in X$ and set $\beta:=\mu_{\mathfrak{p}}(x)$. The following are equivalent:

(i) $\beta_{X}(x)=0$;

(ii) $d \mu_{\mathfrak{p}}^{\beta}(x)=0$;

(iii) $d \eta_{\mathfrak{p}}(x)=0$.

Let $x \in X$ and let $\beta \in \mathfrak{p}_{x}:=\left\{\beta \in \mathfrak{p}: \beta_{X}(x)=0\right\}$. Differentiating the action of $G_{x}$ on $T_{x}(X)$ gives rise to a linear action of $\mathfrak{g}_{x}$ on $T_{x}(X)$. Since elements of $i \mathfrak{u}_{x}$ act as selfadjoint operators on $T_{x}(Z)$ relative to the induced Riemannian structure, our element $\beta$ acts on $T_{x}(X)$ as a selfadjoint operator $d \beta_{X}(x)$ and has real eigenvalues. 


\section{P. Heinzner, G. W. Schwarz and H. Stötzel}

Proposition 2.5. Let $v \in T_{x}(X)$ be an eigenvector of $\beta \in \mathfrak{p}_{x}$ with eigenvalue $\lambda(\beta)$. Let $\gamma(t)$ be a smooth curve in $X$ with $\gamma(0)=x$ and $\frac{d}{d t} \gamma(0)=v$. Then:

(i) $\frac{d^{2}}{d t^{2}}\left(\mu_{\mathfrak{p}}^{\beta} \circ \gamma\right)(0)=\lambda(\beta)\|v\|^{2}$;

(ii) If $x$ is a critical point of $\eta_{\mathfrak{p}}$ and $\beta:=\mu_{\mathfrak{p}}(x)$, then

$$
\frac{d^{2}}{d t^{2}}\left(\eta_{\mathfrak{p}} \circ \gamma\right)(0)=\lambda(\beta)\|v\|^{2}+\left\|d \mu_{\mathfrak{p}}(x)(v)\right\|^{2} .
$$

Proof. We have

$$
\frac{d}{d t} \mu_{\mathfrak{p}}^{\beta}(\gamma(t))=\left(\frac{d}{d t} \gamma(t), \beta_{X}(\gamma(t))\right)_{\gamma(t)}
$$

where $(\cdot, \cdot)_{z}$ denotes the inner product on $T_{z}(X)$ at $z \in X$. There is a neighborhood $U$ of $x \in X$ and a local diffeomorphism $\Psi: U \rightarrow T_{x}(X)$ where $\Psi(x)=0$ and $d \Psi(x)=$ Id. Using the local coordinates given by $\Psi$ we have $(\cdot, \cdot)_{\gamma(t)}=(\cdot, \cdot)_{x}+t(\cdot, \cdot)_{t}$ where $(\cdot, \cdot)_{t}$ is a bilinear form on $T_{x}(X)$ depending smoothly on $t$. From $\beta_{X}(\gamma(t))=t \cdot d \beta_{X}(x) \cdot \frac{d}{d t} \gamma(0)+t^{2} R_{0}(t)=t \lambda(\beta) v+t^{2} R_{0}(t)$, where $R_{0}(t)$ is smooth, we obtain

$$
\begin{aligned}
\frac{d}{d t}\left(\mu_{\mathfrak{p}}^{\beta} \circ \gamma\right)(t) & =\left(\frac{d}{d t} \gamma(t), \beta_{X}(\gamma(t))\right)_{x}+t\left(\frac{d}{d t} \gamma(t), \beta_{X}(\gamma(t))\right)_{t} \\
& =\left(\frac{d}{d t} \gamma(t), t \lambda(\beta) v+t^{2} R_{0}(t)\right)_{x}+t\left(\frac{d}{d t} \gamma(t), t \lambda(\beta) v+t^{2} R_{0}(t)\right)_{t} \\
& =\left(\frac{d}{d t} \gamma(t), t \lambda(\beta) v\right)_{x}+t^{2} R_{1}(t) .
\end{aligned}
$$

This implies part (i).

If $\beta$ is as in part (ii), then $\mu_{\mathfrak{p}}(\gamma(t))=\beta+t \cdot d \mu_{\mathfrak{p}}(x) \cdot v+t^{2} R_{2}(t)$. Hence,

$$
\begin{aligned}
\frac{d}{d t}\left(\eta_{\mathfrak{p}} \circ \gamma\right)(t) & =\left\langle d \mu_{\mathfrak{p}}(\gamma(t)) \frac{d}{d t} \gamma(t), \mu_{\mathfrak{p}}(\gamma(t))\right\rangle \\
& =\frac{d}{d t}\left(\mu_{\mathfrak{p}}^{\beta} \circ \gamma\right)(t)+t\left\langle d \mu_{\mathfrak{p}}(\gamma(t)) \frac{d}{d t} \gamma(t), d \mu_{\mathfrak{p}}(x) \cdot v\right\rangle+t^{2} R_{3}(t)
\end{aligned}
$$

and part (ii) follows.

If $\mathfrak{m}$ is a subspace of a Lie algebra $\mathfrak{l}$ and $\beta \in \mathfrak{l}$, set $\mathfrak{m}^{\beta}:=\{\xi \in \mathfrak{m}:[\xi, \beta]=0\}$.

Lemma 2.6. Let $\beta:=\mu_{\mathfrak{p}}(x)$ and assume that $\beta \in \mathfrak{p}_{x}$. Let $\zeta \in \mathfrak{k}$. If $\zeta_{X}(x)$ is the sum of positive eigenvectors of $\beta$, then $\zeta \in \mathfrak{k}^{\beta}$.

Proof. Let $\gamma(t):=\exp t \zeta \cdot x$. Proposition 2.5 implies that $0 \leqslant \frac{d^{2}}{d t^{2}}\left(\mu_{\mathfrak{p}}^{\beta} \circ \gamma\right)(0)$. Since $\mu_{\mathfrak{p}}$ is $K$-equivariant and the $K$-action on $\mathfrak{p}$ is by linear isometries we have

$$
\frac{d^{2}}{d t^{2}}\left(\mu_{\mathfrak{p}}^{\beta} \circ \gamma\right)(0)=\left.\frac{d^{2}}{d t^{2}}\right|_{0}\left\langle\exp (t \zeta) \cdot \mu_{\mathfrak{p}}(x), \beta\right\rangle=\langle[\zeta,[\zeta, \beta]], \beta\rangle=-\|[\zeta, \beta]\|^{2} \leqslant 0 .
$$

This shows that $[\zeta, \beta]=0$, i.e. $\zeta \in \mathfrak{k}^{\beta}$.

\section{The slice and quotient theorems}

In this section we recall for the convenience of the reader some results from [HS07a, HS07b]. For any Lie group $G$, a closed subgroup $H$ and any set $S$ with an $H$-action we denote by $G \times{ }^{H} S$ the $G$-bundle over $G / H$ associated with the $H$-principal bundle $G \rightarrow G / H$. This is the orbit space of 


\section{STRATIFICATIONS}

the $H$-action on $G \times S$ given by $h \cdot(g, s)=\left(g h^{-1}, h \cdot s\right)$ where $g \in G, s \in S$ and $h \in H$. The $H$-orbit of $(g, s)$, considered as a point in $G \times{ }^{H} S$, is denoted by $[g, s]$.

Let $G=K \exp \mathfrak{p}$ be a compatible closed subgroup of $U^{\mathbb{C}}$. For $\beta \in \mathfrak{p}$ set $\mathcal{M}_{\mathfrak{p}}(\beta):=\mu_{\mathfrak{p}}^{-1}(\beta) \subset X$ and set $\mathcal{M}_{\mathfrak{p}}:=\mathcal{M}_{\mathfrak{p}}(0)$. Let $x \in \mathcal{M}_{\mathfrak{p}}$. Then $G_{x}=K_{x} \exp \mathfrak{p}_{x}$ (see [HS07a, Lemma 5.5]). Since the $G_{x}$-representation on $T_{x}(X)$ is completely reducible [HS07a, Corollary 14.9], there is a $G_{x}$-stable decomposition $T_{x}(X)=\mathfrak{g} \cdot x \oplus W$. Now the slice theorem for $Z$ (see [HS07a, Theorems 14.10 and 14.21]) pulls back to the following slice theorem for $X$.

Theorem 3.1 (Slice Theorem). Let $x \in \mathcal{M}_{\mathfrak{p}}$. Then there exists a $G_{x}$-stable open neighborhood $S$ of $0 \in W$, a $G$-stable open neighborhood $\Omega$ of $x \in X$ and a $G$-equivariant diffeomorphism $\Psi: G \times{ }^{G_{x}} S \rightarrow \Omega$ where $\Psi([e, 0])=x$.

Actually, we have a slice theorem at every $x \in X$. Set $\beta:=\mu_{\mathfrak{p}}(x)$ and let $G^{\beta}=\{g \in G$ : Ad $g \cdot \beta=\beta$ \} denote the centralizer of $\beta$. Then we have a slice for the action of $G^{\beta}$, as follows.

The centralizer $G^{\beta}$ is a compatible subgroup of $U^{\mathbb{C}}$ with Cartan decomposition $G^{\beta}=K^{\beta} \exp \left(\mathfrak{p}^{\beta}\right)$ where $K^{\beta}=K \cap G^{\beta}$ and $\mathfrak{p}^{\beta}=\{\xi \in \mathfrak{p}: \operatorname{ad}(\xi) \beta=0\}$. The group $G^{\beta}$ is also compatible with the Cartan decomposition of $\left(U^{\mathbb{C}}\right)^{\beta}=\left(U^{\beta}\right)^{\mathbb{C}}$ and $\beta$ is fixed by the action of $U^{\beta}$ on $\mathfrak{u}^{\beta}$. This implies that the $\mathfrak{u}^{\beta}$-component of $\mu$ defines a $U^{\beta}$-equivariant shifted gradient map $\widehat{\mu_{\mathfrak{u}^{\beta}}}: Z \rightarrow \mathfrak{u}^{\beta}, \widehat{\mu_{\mathfrak{u}^{\beta}}}(z)=\mu_{\mathfrak{u}^{\beta}}(z)-\beta$. The associated $G^{\beta}$-gradient map is given by $\widehat{\mu_{\mathfrak{p}^{\beta}}}: X \rightarrow \mathfrak{p}^{\beta}, \widehat{\mu_{\mathfrak{p}^{\beta}}}(z)=\mu_{\mathfrak{p}^{\beta}}(z)-\beta$. This shows that the slice theorem applies to the action of $G^{\beta}$ at every point $\left.x \in \widehat{\left(\mu_{\mathfrak{p}^{\beta}}\right.}\right)^{-1}(0)=\mathcal{M}_{\mathfrak{p}^{\beta}}(\beta)$. In particular, if $G$ is commutative, then we have a slice theorem for $G$ at every point of $X$.

For $H$ a subgroup of $G, M$ a subset of $Z$ and $Y$ an $H$-stable subset of $Z$, we define the saturation $\mathcal{S}_{H}(M)(Y)$ to be $\{z \in Y: \overline{H \cdot z} \cap M \neq \emptyset\}$. Here $\overline{H \cdot z}$ denotes the closure of $H \cdot z$ in $Y$. In general, $\mathcal{S}_{H}(M)(Y)$ is a proper subset of $\mathcal{S}_{H}(M)(Z) \cap Y$. However, in the case where $Y$ is closed in $Z$ these sets agree. The set $\mathcal{S}_{G}\left(\mathcal{M}_{\mathfrak{p}}\right)(Y)$ is called the set of semistable points of $Y$ with respect to $\mu_{\mathfrak{p}}$. In [HS07b] it has been shown that $\mathcal{S}_{G}\left(\mathcal{M}_{\mathfrak{p}}(\beta)\right)(Z)$ is open in $Z$ for every $\beta \in \mathfrak{p}$. For a closed $G$-stable subset $Y$ of $Z$ this implies that $\mathcal{S}_{G}\left(\mathcal{M}_{\mathfrak{p}}(\beta)\right)(Y)$ is open in $Y$. Inspecting the proof in [HS07b] one can more generally show that $\mathcal{S}_{G}\left(\mathcal{M}_{\mathfrak{p}}(\beta)\right)(X)$ is open in $X$ for any locally closed $G$-stable submanifold $X$ of $Z$. Since we have fixed $X$, we usually write $\mathcal{S}_{G}\left(\mathcal{M}_{\mathfrak{p}}(\beta)\right)$ for $\mathcal{S}_{G}\left(\mathcal{M}_{\mathfrak{p}}(\beta)\right)(X)$. The set of semistable points plays a major role in $[\mathrm{HS} 07 \mathrm{a}]$. One reason for this is the quotient theorem which we now formulate.

Let $Y \subset Z$ be $G$-stable and let $x, y \in Y$. We define a relation $\sim$ on $Y$ where $x \sim y$ if and only if $Y \cap \overline{G \cdot x} \cap \overline{G \cdot y} \neq \emptyset$. If this relation is in fact an equivalence relation we denote the corresponding quotient by $Y / / G$ and call it the topological Hilbert quotient of $Y$ by the action of $G$.

Theorem 3.2 (Quotient Theorem [HS07a]). Assume that $X=\mathcal{S}_{G}\left(\mathcal{M}_{\mathfrak{p}}\right)$. Then the topological Hilbert quotient $X / / G$ exists and has the following properties.

(i) Every fiber of $\pi$ contains a unique closed G-orbit. Any other orbit in the fiber has strictly larger dimension.

(ii) The closure of every $G$-orbit in a fiber of $\pi$ contains the closed G-orbit.

(iii) Every fiber of $\pi$ intersects $\mathcal{M}_{\mathfrak{p}}$ in a unique $K$-orbit which lies in the unique closed $G$-orbit.

(iv) The inclusion $\mathcal{M}_{\mathfrak{p}} \hookrightarrow X$ induces a homeomorphism $\mathcal{M}_{\mathfrak{p}} / K \cong X / / G$.

As in the case of the slice theorem, we have local versions of the quotient theorem. For any $\beta \in \mathfrak{p}$ we have the open subset of semistable points $\mathcal{S}_{G^{\beta}}\left(\mathcal{M}_{\mathfrak{p}^{\beta}}(\beta)\right)$ in $X$, and we can apply the quotient theorem for the action of $G^{\beta}$. 


\section{P. Heinzner, G. W. Schwarz and H. Stötzel}

\section{Fixed points and parabolic subgroups}

Let $\beta \in \mathfrak{p}$. We have a vector field $\beta_{G}$ whose one-parameter subgroup is given by $(t, y) \mapsto \exp (t \beta) y$ $\exp (-t \beta)$. Then

$$
G^{\beta}=\left\{y \in G: \beta_{G}(y)=0\right\}=\{y \in G: \exp (t \beta) y \exp (-t \beta)=y \text { for all } t \in \mathbb{R}\} .
$$

We have the parabolic subgroup

$$
G^{\beta_{+}}:=\left\{y \in G: \lim _{t \rightarrow-\infty} \exp (t \beta) y \exp (-t \beta) \text { exists }\right\}
$$

with unipotent radical

$$
R^{\beta_{+}}:=\left\{y \in G: \lim _{t \rightarrow-\infty} \exp (t \beta) y \exp (-t \beta)=e \mid\right\} .
$$

Then $G^{\beta_{+}}$is the semi-direct product of $G^{\beta}$ with $R^{\beta_{+}}$and we have the projection $\pi^{\beta_{+}}: G^{\beta_{+}} \rightarrow G^{\beta}$, $\pi^{\beta_{+}}(y):=\lim _{t \rightarrow-\infty} \exp (t \beta) y \exp (-t \beta)$.

Lemma 4.1. For every $\beta \in \mathfrak{p}$ we have $G=K G^{\beta_{+}}$.

Proof. The adjoint orbit $O$ of $U$ through $\beta \in \mathfrak{i} \mathfrak{u} \cong \mathfrak{u}$ can be considered as a Kähler manifold endowed with a holomorphic $U^{\mathbb{C}}$-action. In particular, $G$-acts on $O$. In [HS07b] it is shown that $G \cdot \beta=K \cdot \beta$. Since $G \cdot \beta \cong G / G^{\beta_{+}}$this proves the claim.

We introduce submanifolds of $X$ analogous to $G^{\beta}$ and $G^{\beta_{+}}$. For $\beta \in \mathfrak{p}$ we have the corresponding vector field $\beta_{X}$ on $X$ whose one-parameter subgroup is given by $(t, y) \mapsto \exp (t \beta) \cdot y$. We have the set

$$
X^{\beta}:=\left\{y \in X: \beta_{X}(y)=0\right\}
$$

which is the set of fixed points $\{y \in X: \exp t \beta \cdot y=y$ for all $t \in \mathbb{R}\}$. The set $X^{\beta}$ is $G^{\beta}$-stable and is a subset of the $G^{\beta+}$-stable set

$$
X^{\beta_{+}}:=\left\{y \in X: \lim _{t \rightarrow-\infty} \exp t \beta \cdot y \text { exists }\right\} .
$$

The map $p^{\beta_{+}}: X^{\beta_{+}} \rightarrow X^{\beta}, p^{\beta_{+}}(y)=\lim _{t \rightarrow-\infty} \exp t \beta \cdot y$ is well defined, $G^{\beta}$-equivariant, surjective and its fibers are $R^{\beta_{+}-\text {stable. }}$

In the following we fix $\beta \in \mathfrak{p},\|\beta\|=1$ and discuss, in the spirit of this paper, the relevant properties of the action of the group $\Gamma:=\exp \gamma$ on $X$ where $\gamma:=\mathbb{R} \beta$. Note that $\Gamma$ is a closed compatible subgroup of $U^{\mathbb{C}}$. As an abstract Lie group $\Gamma$ is just the additive group $\mathbb{R}$. The $\Gamma$ gradient map on $X$ is given by $\mu_{\gamma}(y)=\mu_{\gamma}^{\beta}(y) \beta$ and in the following is identified with $\mu_{\gamma}^{\beta}: X \rightarrow \mathbb{R}$. The isotropy group $\Gamma_{x}$ of $\Gamma$ at every point $x \in X$ is compatible with the Cartan decomposition of $U^{\mathbb{C}}$. Since $\Gamma \cong \mathbb{R}$, we have that either $\Gamma_{x}=\{0\}$ or $\Gamma_{x}=\Gamma$. The slice theorem applied at $x \in X \backslash X^{\beta}$ gives the existence of an open $\Gamma$-stable neighborhood $\Omega$ of $x$, a closed submanifold $S$ of $\Omega$ with $x \in S$ such that the map $\Psi: \Gamma \times S \rightarrow \Omega,(g, s) \mapsto g \cdot s$ is a diffeomorphism.

In the case that $x \in X^{\beta}$, the slice theorem gives a linearization of the $\Gamma$-action near $x$, as follows. The linearized vector field $d \beta_{X}(x)$ acts on $T_{x}(X)$ as a selfadjoint operator, also denoted by $\beta$, and $W:=T_{x}(X)$ is a direct sum of one-dimensional eigenspaces. We define $W^{\beta_{+}}:=W^{\beta} \oplus W^{+}$where $W^{+}$is the sum of the eigenspaces of $\beta$ with positive eigenvalues and $W^{\beta}$ is the zero eigenspace of $\beta$. By the slice theorem there is an open $\Gamma$-stable neighborhood $S$ of zero in $T_{x}(X)$ and a $\Gamma$-equivariant diffeomorphism $\Psi$ which maps $S$ onto an open neighborhood $\Omega$ of $x$ in $X$. Since $S$ is $\Gamma$-stable, it contains $\left(S \cap W^{\beta}\right) \oplus W^{+}$. Now $\Psi$ identifies $S \cap W^{\beta_{+}}$with $\Omega \cap X^{\beta_{+}}$and $S \cap W^{\beta}$ with $\Omega \cap X^{\beta}$. Moreover, $\left(p^{\beta_{+}}\right)^{-1} \Psi\left(S \cap W^{\beta}\right)=\Psi\left(\left(S \cap W^{\beta}\right) \oplus W^{+}\right)$is closed in $\Omega$.

Remark 4.2. The slice theorem applied to $\Gamma$ at $x \in X^{\beta}$ shows that, for $y$ near $x$, the limit $\exp t_{n} \beta \cdot y$ exists for some sequence $t_{n} \rightarrow-\infty$ if and only if $\lim _{t \rightarrow-\infty} \exp t \beta \cdot y=x$. 


\section{STRATIFICATIONS}

We summarize our discussion as follows.

Proposition 4.3. Let $\beta \in \mathfrak{p}, \Gamma=\exp \mathbb{R} \beta$ and let $X^{\beta_{+}}$be as above.

(i) The set $X^{\beta}$ is a closed submanifold of $X$.

(ii) The set $X^{\beta_{+}}$is a locally closed $G^{\beta_{+}}$-stable submanifold of $X$.

(iii) The tangent space of $X^{\beta_{+}}$at $x \in X^{\beta}$ is $W^{\beta_{+}}$.

(iv) The map $p^{\beta_{+}}$is a $G^{\beta}$-equivariant strong deformation retraction.

(v) The map $p^{\beta_{+}}$is $G^{\beta_{+}}$-equivariant where the action of $G^{\beta_{+}}$on $X^{\beta}$ is via the quotient mapping to $G^{\beta}$.

Remark 4.4. In addition to the properties in Proposition 4.3, we have that the map $p^{\beta_{+}}$is a smooth locally trivial fibration which realizes $X^{\beta}$ as the topological Hilbert quotient of $X^{\beta_{+}}$with respect

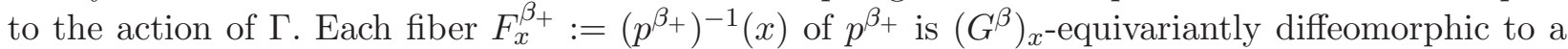
$\left(G^{\beta}\right)_{x^{-}}$-representation $W^{+}(x)$ which, up to isomorphism, depends only on the connected component of $x$ in $X^{\beta}$. Furthermore, the map $G^{\beta} \times^{\left(G^{\beta}\right)_{x}} F_{x}^{\beta_{+}} \rightarrow\left(p^{\beta_{+}}\right)^{-1}\left(G^{\beta} \cdot x\right),[a, z] \mapsto a \cdot z$ is a $G^{\beta}$-equivariant diffeomorphism.

Remark 4.5. If $G=K^{\mathbb{C}}$ for some compact subgroup $K$ of $U$ and if $X$ is a complex submanifold of $Z$, then the manifolds discussed in Proposition 4.3 are complex analytic and the maps are holomorphic.

Of particular interest are the connected components of $X^{\beta}$ on which $\mu_{\mathfrak{p}}^{\beta}=\left\langle\mu_{\mathfrak{p}}, \beta\right\rangle$ is constant. For any $\beta \in \mathfrak{p}$ and $r \in \mathbb{R}$ let $X_{r}^{\beta}:=\left\{y \in X^{\beta}:\left\langle\mu_{\mathfrak{p}}(y), \beta\right\rangle=r\right\}$ and let $X_{r}^{\beta_{+}}:=\left(p^{\beta_{+}}\right)^{-1}\left(X_{r}^{\beta}\right)$.

Proposition 4.6. Let $\beta \in \mathfrak{p}$.

(i) The function $\mu_{\mathfrak{p}}^{\beta}: X^{\beta} \rightarrow \mathbb{R}$ is locally constant. In particular, $X_{r}^{\beta}$ is open and closed in $X^{\beta}$.

(ii) We have $\mu_{\mathfrak{p}}^{\beta}(y) \geqslant r$ for $y \in X_{r}^{\beta_{+}}$and equality holds if and only if $y \in X_{r}^{\beta}$.

Proof. For $v \in T_{y}(X)$ and $y \in X^{\beta}, d \mu_{\mathfrak{p}}^{\beta}(y)(v)=\left(\beta_{X}(y), v\right)=0$. Hence, $\mu_{\mathfrak{p}}^{\beta}$ is locally constant on $X^{\beta}$ and we have part (i).

Let $y \in X_{r}^{\beta_{+}}$. Then $\mu_{\mathfrak{p}}^{\beta}(\exp t \beta \cdot y) \geqslant \lim _{t \rightarrow-\infty} \mu_{\mathfrak{p}}^{\beta}(\exp t \beta \cdot y)=r$. Note that $\mu^{\beta}(y)=r$ if and only if $\exp t \beta \cdot y=y$ for all $t \in \mathbb{R}$ (Lemma 2.1), i.e. $y \in X^{\beta}$. This establishes part (ii).

Remark 4.7. By replacing $\beta$ with $-\beta$ one obtains analogous results for

$$
X^{\beta_{-}}:=\left\{y \in X: \lim _{t \rightarrow+\infty} \exp t \beta \cdot y \text { exists }\right\} .
$$

Let $\beta \in \mathfrak{p}$ and let $\mathfrak{g}^{\beta}=\mathfrak{k}^{\beta}+\mathfrak{p}^{\beta}$ denote the Cartan decomposition of the Lie algebra $\mathfrak{g}^{\beta}$ of the centralizer $G^{\beta}$. For $r \in \mathbb{R}$ let $H_{r}(\beta):=\left\{\zeta \in \mathfrak{p}^{\beta}:\langle\beta, \zeta\rangle=r\right\}$. In the following it is important to note that $H_{r}(\beta)$ is a hyperplane in $\mathfrak{p}^{\beta}$ if and only if $\beta \neq 0$. If $\beta \neq 0$ we have the corresponding half-spaces $H_{r}^{+}(\beta):=\left\{\zeta \in \mathfrak{p}^{\beta}:\langle\beta, \zeta\rangle \geqslant r\right\}$ and $H_{r}^{-}(\beta):=\left\{\zeta \in \mathfrak{p}^{\beta}:\langle\beta, \zeta\rangle \leqslant r\right\}$. These sets are also defined in the case where $\beta=0$ but then they are either empty $(r \neq 0)$ or all of them coincide with $\mathfrak{p}^{\beta}(r=0)$. 
Corollary 4.8. Let $\beta \in \mathfrak{p}$. Then:

(i) $\mu_{\mathfrak{p}^{\beta}}\left(X_{r}^{\beta_{+}}\right) \subset H_{r}^{+}(\beta)$;

(ii) $\mu_{\mathfrak{p}^{\beta}}\left(X_{r}^{\beta_{-}}\right) \subset H_{r}^{-}(\beta)$;

(iii) $X_{r}^{\beta}=X_{r}^{\beta_{-}} \cap X_{r}^{\beta_{+}}=\mu_{\mathfrak{p}^{\beta}}^{-1}\left(H_{r}(\beta)\right) \cap X_{r}^{\beta_{+}}$.

Remark 4.9. For fixed $r$ the manifold $X_{r}^{\beta_{+}}$is defined only in terms of the group $\Gamma:=\exp \mathbb{R} \beta$. This means that $X_{r}^{\beta_{+}}$remains unchanged for any compatible subgroup $G$ of $U^{\mathbb{C}}$ which contains $\Gamma$ and stabilizes $X$. Note that $X_{r}^{\beta}$ is $G^{\beta}$-stable.

\section{Critical points, slices and quotients}

Let $\mu_{\mathfrak{p}^{\beta}}: X \rightarrow \mathfrak{p}^{\beta}$ denote the gradient map associated with the moment map $\mu_{\mathfrak{u}^{\beta}}: Z \rightarrow \mathfrak{u}^{\beta}$ (see $\S 3$ ). Recall that $\mathcal{M}_{\mathfrak{p}^{\beta}}(\beta)$ is the zero fiber of the shifted gradient map $\widehat{\mu_{\mathfrak{p}^{\beta}}}=\mu_{\mathfrak{p}^{\beta}}-\beta$.

For $\beta \in \mathfrak{p}$ we set $S^{\beta_{+}}:=\mathcal{S}_{G^{\beta}}\left(\mathcal{M}_{\mathfrak{p}^{\beta}}(\beta)\right)\left(X_{\|\beta\|^{2}}^{\beta_{+}}\right)$, i.e. $S^{\beta_{+}}$is the set of $G^{\beta}$-semistable points in $X_{\|\beta\|^{2}}^{\beta_{+}}$ with respect to the shifted gradient map $\mu_{\mathfrak{p}^{\beta}}-\beta$. The set $S_{\beta}:=G \cdot S^{\beta_{+}}$is called the pre-stratum associated with $\beta$.

Remark 5.1. The set $S^{\beta_{+}}$is a locally closed submanifold of $X$ since it is an open subset of $X^{\beta_{+}}$.

Remark 5.2. As we already noted (see Remark 4.9), the set $X_{\|\beta\|^{2}}^{\beta_{+}}$only depends on the group $\Gamma=\exp (\mathbb{R} \beta)$. However, in general, $S^{\beta+}$ depends on $G^{\beta}$.

Let $\mathcal{C}_{\mathfrak{p}}$ denote the set of critical points of $\eta_{\mathfrak{p}}: X \rightarrow \mathbb{R}, \eta_{\mathfrak{p}}(x)=\frac{1}{2}\left\|\mu_{\mathfrak{p}}(x)\right\|^{2}$ and set $\mathcal{B}_{\mathfrak{p}}:=\mu_{\mathfrak{p}}\left(\mathcal{C}_{\mathfrak{p}}\right)$. Since $\eta_{\mathfrak{p}}$ is $K$-invariant the sets $\mathcal{C}_{\mathfrak{p}}$ and $\mathcal{B}_{\mathfrak{p}}$ are $K$-stable.

We now formulate our main general results.

Theorem 5.3 (Slice Theorem for Pre-Strata). Let $\beta \in \mathcal{B}_{\mathfrak{p}}$ and let $S_{\beta}$ be the pre-stratum associated with $\beta$.

(i) The pre-stratum $S_{\beta}$ is a locally closed submanifold of $X$ and $S^{\beta_{+}}$is a $G^{\beta_{+}}$-stable locally closed submanifold of $X$.

(ii) The natural map $G \times{ }^{G^{\beta}} S^{\beta_{+}} \rightarrow S_{\beta}$ is a diffeomorphism.

(iii) The natural map $K \times{ }^{K^{\beta}} S^{\beta_{+}} \rightarrow S_{\beta}$ is a diffeomorphism.

The following is an analogue of the quotient Theorem 3.2 for semistable points in the context of pre-strata. Here $\mathcal{M}_{\mathfrak{p}}(K \cdot \beta):=\mu_{\mathfrak{p}}^{-1}(K \cdot \beta)$ plays the role of $\mathcal{M}_{\mathfrak{p}}$ in Theorem 3.2 and the case where $\beta=0$ is just Theorem 3.2 .

Theorem 5.4 (Quotient Theorem for Pre-Strata). Let $\beta \in \mathcal{B}_{\mathfrak{p}}$ and let $S_{\beta}$ be the pre-stratum associated with $\beta$. Then the topological Hilbert quotient $S_{\beta} / / G$ exists. Let $\pi_{S_{\beta}}: S_{\beta} \rightarrow S_{\beta} / / G$ denote the quotient map.

(i) Every fiber of $\pi_{S_{\beta}}$ contains a unique closed G-orbit. Any other orbit in the fiber has strictly larger dimension.

(ii) The closure of every $G$-orbit in a $\pi_{S_{\beta}}$-fiber contains the closed $G$-orbit.

(iii) Every fiber of $\pi_{S_{\beta}}$ intersects $\mathcal{M}_{\mathfrak{p}}(K \cdot \beta) \cap S_{\beta}$ in a unique $K$-orbit which lies in the unique closed $G$-orbit.

(iv) The inclusion $\mathcal{M}_{\mathfrak{p}}(K \cdot \beta) \cap S_{\beta} \hookrightarrow S_{\beta}$ induces a homeomorphism $\left(\mathcal{M}_{\mathfrak{p}}(K \cdot \beta) \cap S_{\beta}\right) / K \cong S_{\beta} / / G$. 


\section{StRATificATions}

Note that we can apply Theorem 3.2 to the set of semistable points $S^{\beta_{+}}=\mathcal{S}_{G^{\beta}}\left(\mathcal{M}_{\mathfrak{p}^{\beta}}(\beta)\right)\left(X_{\|\beta\|^{2}}^{\beta_{+}}\right)$. In the following discussion it will turn out that the quotients $S^{\beta_{+}} / / G^{\beta}$ and $S_{\beta} / / G$ are isomorphic (Proof of Theorem 5.4) and that $\mathcal{M}_{\mathfrak{p}^{\beta}}(\beta) \cap S^{\beta_{+}} \subset \mathcal{M}_{\mathfrak{p}}(K \cdot \beta) \cap S_{\beta}$ (Lemma 5.6 and Proposition 5.8). We may summarize all of this in the following commutative diagram.

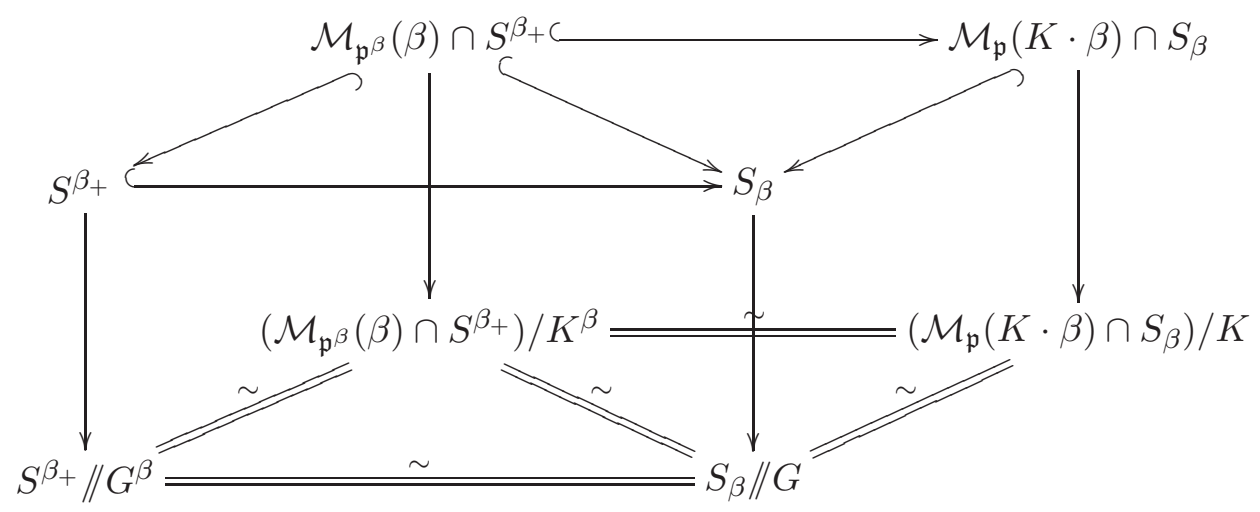

We also see that $\mathcal{M}_{\mathfrak{p}^{\beta}}(\beta) \cap S^{\beta_{+}}$is the set of minima of $\eta_{\mathfrak{p}} \mid S^{\beta_{+}}\left(\right.$Lemma 5.12) and that $\mathcal{M}_{\mathfrak{p}}(K \cdot \beta) \cap S_{\beta}$ is the set of minima of $\eta_{\mathfrak{p}} \mid S_{\beta}$ (Proposition 5.13).

Theorem 5.5 (Pre-Stratification Theorem). Let $\beta, \tilde{\beta} \in \mathcal{B}_{\mathfrak{p}}$.

(i) If $S_{\tilde{\beta}} \cap \overline{S_{\beta}} \neq \emptyset$ and $K \cdot \beta \neq K \cdot \tilde{\beta}$, then $\|\tilde{\beta}\|>\|\beta\|$.

(ii) The following are equivalent:
(a) $K \cdot \beta=K \cdot \tilde{\beta}$;
(b) $S_{\beta}=S_{\tilde{\beta}}$;
(c) $S_{\beta} \cap S_{\tilde{\beta}} \neq \emptyset$.

Now we give additional information about the pre-strata which will lead to the proofs of the theorems.

Lemma 5.6. For any $\beta \in \mathfrak{p}$ we have:

(i) $\mathcal{M}_{\mathfrak{p}}(\beta) \cap X^{\beta}=\mathcal{M}_{\mathfrak{p}}(\beta) \cap \mathcal{C}_{\mathfrak{p}}$;

(ii) $\mathcal{M}_{\mathfrak{p}}(\beta) \cap X^{\beta}=\mathcal{M}_{\mathfrak{p}^{\beta}}(\beta) \cap X^{\beta}$;

(iii) $\mathcal{M}_{\mathfrak{p}^{\beta}}(\beta) \cap X^{\beta}=\mathcal{M}_{\mathfrak{p}^{\beta}}(\beta) \cap X_{\|\beta\|^{2}}^{\beta_{+}}$.

Proof. The first part is a direct consequence of Lemma 2.3. Since $\mu$ is $\exp (\mathrm{i} \mathbb{R} \beta)$-equivariant, on $X^{\beta}$ it takes values in $\left(\mathfrak{u}^{*}\right)^{\beta}$ and $\mu_{\mathfrak{p}}$ takes values in $\mathfrak{p}^{\beta}$. This implies part (ii). Part (iii) follows from Corollary 4.8 .

Remark 5.7. Lemma 5.6 implies that $S^{\beta_{+}}$(equivalently $S_{\beta}$ ) is nonempty if and only if $\beta \in \mathcal{B}_{\mathfrak{p}}$. This is the only reason why Theorems 5.3 and 5.4 are formulated for $\beta \in \mathcal{B}_{\mathfrak{p}}$ and not for $\beta \in \mathfrak{p}$.

As in $\S 4$ we let $p^{\beta_{+}}: X^{\beta_{+}} \rightarrow X^{\beta}$ denote the $G^{\beta}$-equivariant map $p^{\beta_{+}}(y)=\lim _{t \rightarrow-\infty} \exp t \beta \cdot y$.

Proposition 5.8. For every $\beta \in \mathcal{B}_{\mathfrak{p}}$ we have the following:

(i) $S^{\beta_{+}}=\left(p^{\beta_{+}}\right)^{-1}\left(\mathcal{S}_{G^{\beta}}\left(\mathcal{M}_{\mathfrak{p}^{\beta}}(\beta)\right)\left(X^{\beta}\right)\right)$;

(ii) $S^{\beta_{+}}$is $G^{\beta_{+}-\text {stable; }}$

(iii) $\mathcal{M}_{\mathfrak{p}^{\beta}}(\beta) \cap S^{\beta_{+}}=\mathcal{M}_{\mathfrak{p}^{\beta}}(\beta) \cap X^{\beta}$;

(iv) $\mathcal{S}_{G^{\beta}}\left(\mathcal{M}_{\mathfrak{p}^{\beta}}(\beta)\right)\left(X^{\beta}\right)$ is a $G^{\beta}$-equivariant strong deformation retract of $S^{\beta_{+}}$. 


\section{P. Heinzner, G. W. Schwarz and H. Stötzel}

Proof. Let $y \in X^{\beta_{+}}$and set $x=p^{\beta_{+}}(y)$. Since $p^{\beta_{+}}$is $G^{\beta}$-equivariant and fixes $X^{\beta}$ and since $x$ lies in $\overline{G^{\beta} \cdot y}$, we have that $\emptyset \neq \overline{G^{\beta} \cdot x} \cap X^{\beta} \cap \mathcal{M}_{\mathfrak{p}^{\beta}}(\beta)$ if and only if $\emptyset \neq \overline{G^{\beta} \cdot y} \cap X^{\beta} \cap \mathcal{M}_{\mathfrak{p}^{\beta}}(\beta)$. Hence, the equality in part (i) follows from Lemma 5.6. Invariance of $S^{\beta_{+}}$with respect to the $G^{\beta_{+}}$ action follows from parts (i) and (iii) follows from Lemma 5.6(iii). The deformation of $S^{\beta_{+}}$onto $\mathcal{S}_{G^{\beta}}\left(\mathcal{M}_{\mathfrak{p}^{\beta}}(\beta)\right)\left(X^{\beta}\right)$ is given by $(t, y) \mapsto \exp t \beta \cdot y$.

Corollary 5.9. For all $\beta \in \mathcal{B}_{\mathfrak{p}}$ we have $S_{\beta}=K \cdot S^{\beta_{+}}$.

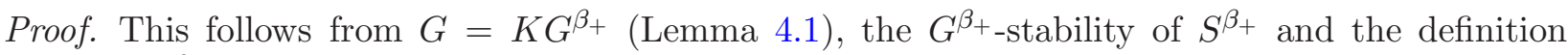
$S_{\beta}=G \cdot S^{\beta_{+}}$.

By Theorem 3.2 the topological Hilbert quotients $\mathcal{S}_{G^{\beta}}\left(\mathcal{M}_{\mathfrak{p}^{\beta}}(\beta)\right)\left(X^{\beta}\right) / / G^{\beta}$ and $S^{\beta_{+}} / / G^{\beta}$ exist. Proposition 5.8 implies that the inclusion $\mathcal{S}_{G^{\beta}}\left(\mathcal{M}_{\mathfrak{p}^{\beta}}(\beta)\right)\left(X^{\beta}\right) \hookrightarrow S^{\beta_{+}}$induces an isomorphism of these quotients. Note that for $x, y \in S^{\beta_{+}}$we have $\overline{G^{\beta} \cdot x} \cap \overline{G^{\beta} \cdot y} \cap S^{\beta_{+}} \neq \emptyset$ if and only if $\overline{G^{\beta_{+}} \cdot x} \cap$ $\overline{G^{\beta_{+}} \cdot y} \cap S^{\beta_{+}} \neq \emptyset$ by Proposition 4.3. Therefore, the quotient $S^{\beta_{+}} / / G^{\beta_{+}}$exists and is not only isomorphic to but also equal to $S^{\beta_{+}} / / G^{\beta}$. We summarize this discussion in the following commutative diagram.

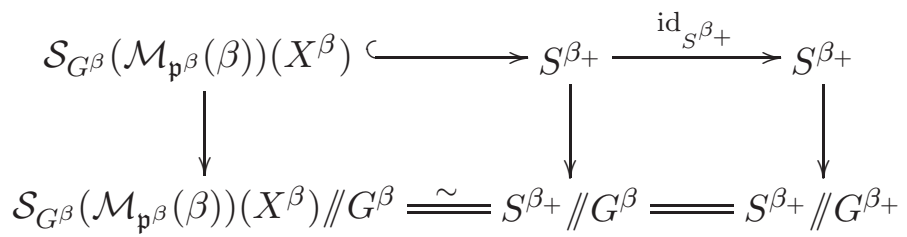

Recall that by Theorem 3.2 each fiber of the quotient $S^{\beta_{+}} \rightarrow S^{\beta_{+}} / / G^{\beta}$ contains a unique closed $G^{\beta}$-orbit which is the unique orbit of minimal dimension in that fiber. We now show the analogous fact for the action of $G^{\beta_{+}}$on $S^{\beta_{+}}$. We make use of the following remark.

Remark 5.10. Let $H$ be a Lie group acting on a manifold $Y$. Then for all $d \in \mathbb{N},\{y \in Y \mid \operatorname{dim} H \cdot y$ $\geqslant d\}$ is open.

Proposition 5.11. Let $G^{\beta} \cdot x$ be the unique closed $G^{\beta}$-orbit in a fiber $F$ of the quotient $S^{\beta_{+}} \rightarrow$

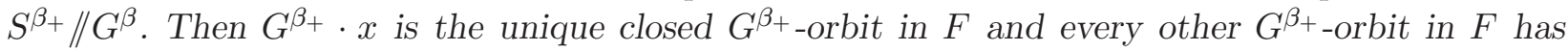
strictly larger dimension.

Proof. Since $p^{\beta_{+}}(x) \in G^{\beta} \cdot x$, we must have that $x \in X^{\beta}$. To simplify notation let $Q$ denote $G^{\beta_{+}}$. Let $z \in F$ and set $y:=p^{\beta+}(z)$. Then $y \in F \cap X^{\beta}$.

Assume that $Q \cdot z \neq Q \cdot y$. We show that $\operatorname{dim} Q \cdot z>\operatorname{dim} Q \cdot y$. Let $\mathfrak{q}_{y}^{c}$ be a subspace of $\mathfrak{q}$ complementary to $\mathfrak{q}_{y}$. Since an $\exp (\mathbb{R} \beta)$-invariant neighborhood of $y$ in $X^{\beta_{+}}$can be identified with an $\exp (\mathbb{R} \beta)$-invariant neighborhood of 0 in the tangent space $T_{y}\left(X^{\beta_{+}}\right)$(see $\S 4$ ) and since $\mathfrak{q}_{y}^{c} \cdot y=\mathfrak{q} \cdot y$ is $\exp (\mathbb{R} \beta)$-stable, there is an $\exp (\mathbb{R} \beta)$-invariant locally closed submanifold $N_{y}$ of $X^{\beta_{+}}$with $y \in N_{y}$ and $T_{y}\left(X^{\beta_{+}}\right)=\mathfrak{q}_{y}^{c} \cdot y \oplus T_{y}\left(N_{y}\right)$. This implies that the map $\Phi_{y}: \mathfrak{q}_{y}^{c} \times N_{y} \rightarrow X^{\beta_{+}},(\xi, w) \mapsto \exp (\xi) \cdot w$, is a local diffeomorphism near $(0, y)$. Since $Q \cdot z$ intersects every open neighborhood of $y$ and since $\exp \left(\mathfrak{q}_{y}^{c}\right) \subset Q$, we may assume that there is a $z^{\prime} \in Q \cdot z$ such that $z^{\prime} \in N_{y}$ and such that $\operatorname{dim} \mathfrak{q}_{y}^{c} \cdot z^{\prime}=\operatorname{dim} \mathfrak{q}_{y}^{c} \cdot y$. Since $Q \cdot z \neq Q \cdot y, z^{\prime} \notin X^{\beta}$ and $0 \neq \beta_{X}\left(z^{\prime}\right) \in T_{z^{\prime}}\left(N_{y}\right)$ does not lie in $\mathfrak{q}_{y}^{c} \cdot z^{\prime}$. Thus, $\operatorname{dim} Q \cdot z^{\prime} \geqslant \operatorname{dim} \mathfrak{q}_{y}^{c}+1>\operatorname{dim} Q \cdot y$.

It now suffices to show that $\operatorname{dim} Q \cdot y>\operatorname{dim} Q \cdot x$ if $Q \cdot y \neq Q \cdot x$. We have that $\operatorname{dim} G^{\beta} \cdot x<\operatorname{dim} G^{\beta} \cdot y$. Since $p^{\beta_{+}}(x)=x$ the $G^{\beta}$-equivariance and $R^{\beta_{+}}$-invariance of $p^{\beta_{+}}$imply that $\operatorname{dim} Q \cdot x=\operatorname{dim} G^{\beta} \cdot x+$ $\operatorname{dim} R^{\beta_{+}} \cdot x$ and similarly for $y$. Since we may assume that $y$ is arbitrarily close to $x$, we have that $\operatorname{dim} R^{\beta_{+}} \cdot y \geqslant \operatorname{dim} R^{\beta_{+}} \cdot x$. Thus, $\operatorname{dim} Q \cdot y=\operatorname{dim} G^{\beta} \cdot y+\operatorname{dim} R^{\beta_{+}} \cdot y>\operatorname{dim} G^{\beta} \cdot x+\operatorname{dim} R^{\beta_{+}} \cdot x=\operatorname{dim} Q \cdot x$.

We have shown that the orbit $Q \cdot x$ is of minimum dimension in $F$. It remains to show that it is the unique closed orbit. Suppose that $x^{\prime} \in \overline{Q \cdot x}$ where $Q \cdot x^{\prime} \neq Q \cdot x$. Then $\operatorname{dim} Q \cdot x \geqslant \operatorname{dim} Q \cdot x^{\prime}$. 


\section{STRATIFICATIONS}

However, this contradicts the fact that $\operatorname{dim} Q \cdot x<\operatorname{dim} Q \cdot x^{\prime}$. Hence, $Q \cdot x$ is closed. By the definition of topological Hilbert quotient there are no other closed orbits in the fiber $F$.

Lemma 5.12. Let $\beta \in \mathcal{B}_{\mathfrak{p}}$ and $z \in \overline{S^{\beta_{+}}}$. Then $\eta_{\mathfrak{p}}(z) \geqslant \frac{1}{2}\|\beta\|^{2}$ and equality holds if and only if $z \in \mathcal{M}_{\mathfrak{p}}(\beta) \cap X^{\beta}$.

Proof. We have $\left\|\mu_{\mathfrak{p}}(z)\right\| \geqslant\left\|\mu_{\mathfrak{p} \beta}(z)\right\| \geqslant\|\beta\|$ by Corollary 4.8. If equality holds then $\mu_{\mathfrak{p} \beta}(z)=\beta$ and $z$ is a minimum of $\eta_{\mathfrak{p}^{\beta}} \mid G^{\beta} z$. Therefore, it is a critical point of $\eta_{\mathfrak{p}^{\beta}}$ and we have $z \in \mathcal{M}_{\mathfrak{p}^{\beta}}(\beta) \cap X^{\beta}=$ $\mathcal{M}_{\mathfrak{p}}(\beta) \cap X^{\beta}$ by Corollary 2.4 .

Proposition 5.13. Let $\beta \in \mathcal{B}_{\mathfrak{p}}$ and let $Y_{\beta}$ be a $G$-stable closed subset of $S_{\beta}$ and $\overline{Y_{\beta}}$ its closure in $X$. We have $\eta_{\mathfrak{p}} \mid \overline{Y_{\beta}} \geqslant \frac{1}{2}\|\beta\|^{2}$ and the set of $z \in \overline{Y_{\beta}}$ where $\eta_{\mathfrak{p}}(z)=\frac{1}{2}\|\beta\|^{2}$ coincides with $\mathcal{M}_{\mathfrak{p}}(K \cdot \beta) \cap Y_{\beta}$ and is non-empty if $Y_{\beta}$ is non-empty.

Proof. We have $\overline{Y_{\beta}}=K \cdot \overline{Y^{\beta_{+}}}$where $Y^{\beta_{+}}:=Y_{\beta} \cap S^{\beta_{+}}$(Corollary 5.9). For $z \in \overline{Y_{\beta}}$ we have $z=k \cdot y$ where $k \in K$ and $y \in \overline{Y^{\beta_{+}}} \subset \overline{S^{\beta_{+}}}$. Then $\left\|\mu_{\mathfrak{p}}(z)\right\|=\left\|\mu_{\mathfrak{p}}(y)\right\| \geqslant\|\beta\|$. If we have equality then $\mu_{\mathfrak{p}^{\beta}}(y)=\beta$ and $y \in X^{\beta}$ (Lemma 5.12). Now $Y^{\beta_{+}}$is closed in $S^{\beta_{+}}$. Therefore, $y \in \mathcal{M}_{\mathfrak{p}}(\beta) \cap Y^{\beta_{+}}$and $z \in \mathcal{M}_{\mathfrak{p}}(K \cdot \beta) \cap Y_{\beta}$.

Finally, $\mathcal{M}_{\mathfrak{p}}(K \cdot \beta) \cap Y_{\beta}$ is non-empty since $\overline{G^{\beta} \cdot y}$ intersects $\mathcal{M}_{\mathfrak{p}^{\beta}}(\beta) \cap X^{\beta}$ for every $y \in Y^{\beta_{+}} \subset$ $S^{\beta_{+}}$.

Corollary 5.14. The closure of every $G$-orbit in $S_{\beta}$ intersects $\mathcal{M}_{\mathfrak{p}}(\beta) \cap X^{\beta}$.

We now work towards the proof of the slice theorem for pre-strata.

Lemma 5.15. Let $\beta \in \mathcal{B}_{\mathfrak{p}}$. The identity $S^{\beta_{+}} \rightarrow S^{\beta_{+}}$induces a diffeomorphism $\phi: K \times{ }^{K^{\beta}} S^{\beta_{+}} \rightarrow$ $G \times{ }^{\beta^{\beta}} S^{\beta_{+}}$.

Proof. Since $G=K G^{\beta_{+}}, K \cap G^{\beta_{+}}=K^{\beta}$ and $\mathfrak{g}=\mathfrak{k}+\mathfrak{g}^{\beta_{+}}, \phi$ is a one-to-one onto submersion between manifolds of the same dimension, hence a diffeomorphism.

In the following we fix $\beta \in \mathcal{B}_{\mathfrak{p}}$ and define $\Psi: G \times{ }^{G^{\beta_{+}}} S^{\beta_{+}} \rightarrow S_{\beta}$ by $\Psi([g, s])=g \cdot s$.

Lemma 5.16. The map $\Psi$ is an immersion.

Proof. First we show that $\Psi$ is an immersion at any $x \in \mathcal{M}_{\mathfrak{p}}(\beta) \cap S^{\beta_{+}}$. Since $\mathfrak{g}=\mathfrak{k}+\mathfrak{g}^{\beta_{+}}$and $\mathfrak{g}^{\beta_{+}} \cdot x \subset T_{x}\left(S^{\beta_{+}}\right)$it is sufficient to show that $\zeta \in \mathfrak{k}$ and $\zeta_{X}(x) \in T_{x}\left(S^{\beta_{+}}\right)$implies that $\zeta \in \mathfrak{k}^{\beta} \subset \mathfrak{g}^{\beta_{+}}$. This is a consequence of Lemma 2.6 since $T_{x}\left(S^{\beta_{+}}\right)$is the sum of the eigenspaces with non-negative eigenvalues of the isotropy action of $\beta$ on $T_{x}(X)$. Since every $G^{\beta}$-orbit in $S^{\beta_{+}}$intersects every open neighborhood of $\mathcal{M}_{\mathfrak{p}}(\beta) \cap S^{\beta_{+}}$and $\Psi$ is $G$-equivariant it follows that $\Psi$ is an immersion.

Proposition 5.17. Let $w_{n} \in G \times{ }^{G^{\beta_{+}}} S^{\beta_{+}}$be a sequence such that $\Psi\left(w_{n}\right)$ converges to $x \in$ $\mathcal{M}_{\mathfrak{p}}(\beta) \cap X^{\beta}$. Then the sequence $w_{n}$ has a convergent subsequence and every convergent subsequence converges to $[e, x]$.

Proof. Let $w_{n}=\left[k_{n}, s_{n}\right]$ where $k_{n} \in K$ and $s_{n} \in S^{\beta_{+}}$(Lemma 5.15). We may assume that $k_{n}$ converges to $k \in K$. This implies that $\lim _{n \rightarrow \infty} \Psi\left(k_{n}^{-1}\left[k_{n}, s_{n}\right]\right)=\lim _{n \rightarrow \infty} s_{n}=k^{-1} \cdot x \in \overline{S^{\beta_{+}}} \subset \overline{X_{\|\beta\|^{2}}^{\beta_{+}}}$. From $\left\|\mu_{\mathfrak{p}}\left(k^{-1} \cdot x\right)\right\|=\left\|\mu_{\mathfrak{p}}(x)\right\|=\|\beta\|$ and $k^{-1} \cdot x \in \overline{S^{\beta_{+}}}$we obtain that $k^{-1} x \in \mathcal{M}_{\mathfrak{p}}(\beta) \cap X^{\beta}$ (Lemma 5.12). Since also $x \in \mathcal{M}_{\mathfrak{p}}(\beta) \cap X^{\beta}$ we conclude that $k \in K^{\beta}$ and that $\lim _{n \rightarrow \infty}\left[k_{n}, s_{n}\right]=$ $\left[k, k^{-1} \cdot x\right]=[e, x]$.

Corollary 5.18. The map $\Psi$ is injective. 


\section{P. Heinzner, G. W. Schwarz and H. Stötzel}

Proof. We first show that for every $x \in \mathcal{M}_{\mathfrak{p}}(\beta) \cap X^{\beta}$ there exists an open neighborhood $\Omega$ of $x \in X$ such that $\Psi \mid \Psi^{-1}\left(S_{\beta} \cap \Omega\right)$ is injective. If $\Psi \mid \Psi^{-1}\left(S_{\beta} \cap \Omega\right)$ is not injective for $\Omega$ sufficiently small, then there are $v_{n}, w_{n} \in G \times{ }^{G^{\beta}+} S^{\beta_{+}}$such that $v_{n} \neq w_{n}, \Psi\left(v_{n}\right)=\Psi\left(w_{n}\right)$ and $\lim _{n \rightarrow \infty} \Psi\left(v_{n}\right)=x$. Since $\Psi$ is an immersion (Lemma 5.16) its restriction to some open neighborhood $W$ of $[e, x]$ in $G \times{ }^{G^{\beta}}+S^{\beta_{+}}$is injective. We may assume that $\lim _{n \rightarrow \infty} v_{n}=\lim _{n \rightarrow \infty} w_{n}=[e, x]$ (Proposition 5.17). This contradicts the injectivity of $\Psi \mid W$.

Since every $G$-orbit in $S_{\beta}$ intersects each neighborhood of a point of $\mathcal{M}_{\mathfrak{p}}(\beta) \cap X^{\beta}$ (Corollary 5.14) and since $\Psi$ is $G$-equivariant, every $G$-orbit in $S_{\beta}$ intersects an open subset $\Omega$ of $X$ non-trivially such that $\Psi \mid \Psi^{-1}\left(S_{\beta} \cap \Omega\right)$ is injective. Hence, $\Psi$ is injective.

Proof of Theorem 5.3 (Slice Theorem for Pre-Strata). We first show part (i). It follows from Proposition 4.3 that $S^{\beta_{+}}$is a locally closed submanifold of $X$. Let $x \in \mathcal{M}_{\mathfrak{p}}(\beta) \cap X^{\beta}$ and choose an open neighborhood $W_{x}$ of $[e, x] \in G \times{ }^{G^{\beta}+} S^{\beta_{+}}$such that $\Psi(W)$ is a submanifold of $X$ (Lemma 5.16). Then there is an open neighborhood $\Omega_{x}$ of $x$ in $X$ such that $\Omega_{x} \cap S_{\beta} \subset \Psi(W)$ since otherwise we could use Proposition 5.17 to arrive at a contradiction. The open sets $g \cdot \Omega_{x}$ cover $S_{\beta}$ (Corollary 5.14) and the open sets $g \cdot W_{x}$ cover $G \times{ }^{G^{\beta_{+}}} S^{\beta_{+}}$where $g \in G, x \in \mathcal{M}_{\mathfrak{p}}(\beta) \cap X^{\beta}$. Since $g \cdot \Omega_{x} \cap S_{\beta} \subset \Psi\left(g \cdot W_{x}\right)=g \cdot \Psi\left(W_{x}\right)$ we have (i).

Part (ii) follows from part (i), Lemma 5.16 and Corollary 5.18. Lemma 5.15 and part (ii) imply part (iii).

Proof of Theorem 5.4 (Quotient Theorem for Pre-Strata). Recall that the topological Hilbert quotients $S^{\beta_{+}} / / G^{\beta}$ and $S^{\beta_{+}} / / G^{\beta_{+}}$exist and coincide. Theorem 5.3 (the slice theorem for pre-strata) implies that $S_{\beta} / / G$ exists and that the inclusion $S^{\beta_{+}} \subset S_{\beta}$ induces an isomorphism $S^{\beta_{+}} / / G^{\beta_{+}} \cong S_{\beta} / / G$.

By Proposition 5.11 every fiber of the quotient map $\pi_{S^{\beta_{+}}}: S^{\beta_{+}} \rightarrow S^{\beta_{+}} / / G^{\beta_{+}}$contains a unique closed $G^{\beta_{+}-\text {orbit }} G^{\beta_{+}} \cdot x$ and every other orbit in that fiber has strictly larger dimension. Assertion (i) then follows from Theorem 5.3 (the slice theorem for pre-strata).

Assertion (ii) follows from the definition of the topological Hilbert quotient and assertion (i).

Let $q \in S_{\beta} / / G$ and set $F_{q}:=\left(\pi_{S_{\beta}}\right)^{-1}(q)$. Then $F_{q} \cap S^{\beta_{+}}$is a fiber of $\pi_{S^{\beta_{+}}}$and intersects $\mathcal{M}_{\mathfrak{p}^{\beta}}(\beta)$ in a unique $K^{\beta}$-orbit $K^{\beta} \cdot x$ where $x \in \mathcal{M}_{\mathfrak{p}^{\beta}}(\beta) \cap X^{\beta}$ and $G^{\beta} \cdot x$ is the unique closed orbit in $F_{q} \cap S^{\beta_{+}}$. Hence, $G \cdot x$ is the unique closed orbit in $F_{q}$ and $K \cdot x$ is the intersection of $F_{q}$ with $\mathcal{M}_{\mathfrak{p}}(K \cdot \beta)$ and we have assertion (iii).

Assertion (iv) follows from assertions (i) and (iii) and Theorem 3.2.

Proof of Theorem 5.5 (Pre-Stratification Theorem). Let $y \in \overline{S_{\beta}} \cap S_{\tilde{\beta}}$. Then $\overline{G \cdot y}$ contains a point $x \in \mathcal{M}_{\mathfrak{p}}(\tilde{\beta})$ (Corollary 5.14 applied to $\tilde{\beta}$ ) and is contained in $\overline{S_{\beta}}$. This implies that $\|\tilde{\beta}\| \geqslant\|\beta\|$ and equality holds if and only if $K \cdot \tilde{\beta}=K \cdot \beta$ (Proposition 5.13). Hence, we must have $\|\tilde{\beta}\|>\|\beta\|$, proving part (i).

We now show part (ii). Assume that part (ii)(a) holds and choose $k \in K$ such that $\tilde{\beta}=k \cdot \beta$. Then $S^{\tilde{\beta}_{+}}=k \cdot S^{\beta_{+}}$implies that $S_{\tilde{\beta}}=S_{\beta}$. Hence, part (ii)(a) implies part (ii)(b). Obviously part (ii)(b) implies part (ii)(c). Assume that part (ii)(c) holds and let $z \in S_{\beta} \cap S_{\tilde{\beta}}$. If part (ii)(a) fails, then part (i) shows that $\|\tilde{\beta}\|>\|\beta\|$ and that $\|\beta\|>\|\tilde{\beta}\|$, a contradiction.

\section{Consequences and special cases}

In this section we point out special cases and several consequences of the results obtained in the previous section. 


\section{STRATIFiCATions}

First note that for $\beta=0$ the pre-stratum $S_{\beta}$ coincides with $S^{\beta_{+}}$and is the set of semistable points $\mathcal{S}_{G}\left(\mathcal{M}_{\mathfrak{p}}\right)$. Theorem 5.3 (the pre-strata slice theorem) is trivial in this case and Theorem 5.4 (the quotient theorem) is just Theorem 3.2. If $G=G^{\beta}$, in particular if $G$ is commutative, then $S_{\beta}=S^{\beta_{+}}$coincides with the set of semistable points $\mathcal{S}_{G}\left(\mathcal{M}_{\mathfrak{p}}(\beta)\right)\left(X_{\|\beta\|^{2}}^{\beta_{+}}\right)$.

For Morse theoretical considerations the following should be noted.

Lemma 6.1. Let $\beta \in \mathcal{B}_{\mathfrak{p}}$. Then $\mathcal{C}_{\mathfrak{p}} \cap S_{\beta}=\mathcal{M}_{\mathfrak{p}}(K \cdot \beta) \cap S_{\beta}$.

Proof. We have $\mathcal{M}_{\mathfrak{p}}(K \cdot \beta) \cap S_{\beta}=K \cdot\left(\mathcal{M}_{\mathfrak{p}}(\beta) \cap X^{\beta}\right) \subset \mathcal{C}_{\mathfrak{p}}$ by Corollary 2.4. Conversely, if $x \in \mathcal{C}_{\mathfrak{p}} \cap S_{\beta}$, let $\tilde{\beta}:=\mu_{\mathfrak{p}}(x)$. Then $x \in \mathcal{M}_{\mathfrak{p}}(\tilde{\beta}) \cap X^{\tilde{\beta}} \subset S_{\tilde{\beta}}$. Since $x \in S_{\beta} \cap S_{\tilde{\beta}}$, we have $K \cdot \beta=K \cdot \tilde{\beta}$, and hence $x \in \mathcal{M}_{\mathfrak{p}}(K \cdot \beta)$.

Corollary 6.2. If $X=\mathcal{S}_{G}\left(\mathcal{M}_{\mathfrak{p}}\right)$, then $x \in X$ is a critical point of $\eta_{\mathfrak{p}}$ if and only if $\eta_{\mathfrak{p}}(x)=0$, i.e. the critical set of $\eta_{\mathfrak{p}}$ coincides with $\mathcal{M}_{\mathfrak{p}}$.

Fix $\beta \in \mathcal{B}_{\mathfrak{p}}$. The first two theorems in $\S 5$ contain precise information about the structure of the $G$-action on $S_{\beta}$ and the quotient map $\pi_{S_{\beta}}: S_{\beta} \rightarrow S_{\beta} / / G$. We now wish to apply Theorem 5.3 (the prestrata slice theorem) for the action of $G^{\beta}$ on $S^{\beta_{+}}$. To simplify notation, let us assume that $X=S_{\beta}$

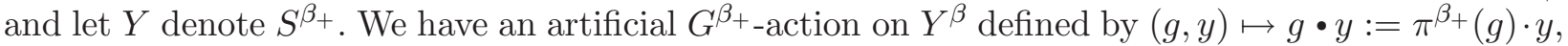
$y \in Y^{\beta}, g \in G^{\beta_{+}}$. The mapping $p^{\beta_{+}}: Y \rightarrow Y^{\beta}$ is equivariant with respect to the given action on $Y$ and the artificial action on $Y^{\beta}$. Here we use the notation introduced in $\S 4$. Note that the $G^{\beta_{+}}$ isotropy group at $y \in Y^{\beta}$ is the semi-direct product $H_{y}:=\left(G^{\beta}\right)_{y} \ltimes R^{\beta_{+}}$. Applying Theorem 5.3 (the slice theorem) we obtain the following.

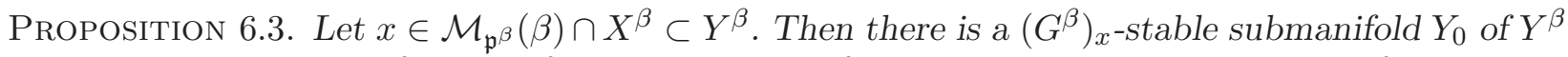
containing $x$ such that $G^{\beta} \cdot Y_{0}=G^{\beta_{+}} \bullet Y_{0}$ is open in $Y^{\beta}$ and such that the natural map $G^{\beta_{+}} \times{ }^{H_{x}} Y_{0} \rightarrow$ $G^{\beta_{+}} \cdot Y_{0}$ is an isomorphism. Let $Y_{1}:=\left(p^{\beta_{+}}\right)^{-1}\left(Y_{0}\right)$. Then $Y_{1}$ is an $H_{x^{-}}$stable submanifold of $Y$,

$$
G^{\beta_{+}} \times{ }^{H_{x}} Y_{1} \rightarrow G^{\beta_{+}} \cdot Y_{1}
$$

is an isomorphism and $G^{\beta_{+}} \cdot Y_{1}$ is open in $Y$. Moreover, $G \cdot Y_{1}$ is open in $X$ and

$$
G \times{ }^{G^{\beta_{+}}}\left(G^{\beta_{+}} \times{ }^{H_{x}} Y_{1}\right) \rightarrow G \cdot Y_{1}
$$

is a $G$-equivariant isomorphism.

Remark 6.4. The entire construction can be carried out in a manner that is compatible with the various Hilbert quotients associated to the $G$-action on $X$. That is, we can arrange that $G^{\beta} \cdot Y_{0}$ is saturated with respect to the quotient map $Y^{\beta} \rightarrow Y^{\beta} / / G^{\beta}$ and that $G^{\beta} \cdot Y_{1}$ is saturated with respect to $Y \rightarrow Y / / G^{\beta}$, etc.

Remark 6.5. The fiber $F$ of $\pi_{G^{\beta}}: Y \rightarrow Y / / G^{\beta}$ through $x \in \mathcal{M}_{\mathfrak{p}^{\beta}}(\beta) \cap Y$ is $G^{\beta}$-isomorphic to $G^{\beta} \times{ }^{\left(G^{\beta}\right)_{x}} F_{x}$ where $F_{x}$ is a closed $\left(G^{\beta}\right)_{x}$-stable subset of the $\left(G^{\beta}\right)_{x}$-stable subspace $W^{\beta_{+}}$(see $\S 4$ ) of $T_{x}(X)$. In this identification every $\left(G^{\beta}\right)_{x}$-orbit in $F_{x}$ contains $0 \in W^{\beta_{+}}$in its closure. Since $F$ is also a fiber of $\pi_{G^{\beta_{+}}}$, the $\left(G^{\beta}\right)_{x}$-space $F_{x}$ is equipped with a topological action of $H_{x}=\left(G^{\beta}\right)_{x} \ltimes R^{\beta_{+}}$.

Before we discuss open pre-strata for general $X$ we need to compute the Hessian of $\eta_{\mathfrak{p}}$ at critical points.

Proposition 6.6. Let $x \in \mathcal{C}_{\mathfrak{p}}$ be a critical point of $\eta_{\mathfrak{p}}: X \rightarrow \mathbb{R}$ and let $S_{\beta}$ be the associated pre-stratum. Let $H_{x}\left(\eta_{\mathfrak{p}}\right)$ denote the Hessian of $\eta_{\mathfrak{p}}$ at $x$. Then:

(i) $H_{x}\left(\eta_{\mathfrak{p}}\right)=0$ on $T_{x}(K \cdot x)$;

(ii) $H_{x}\left(\eta_{\mathfrak{p}}\right)>0$ on $\mathfrak{p}^{\beta} \cdot x+\mathfrak{r}^{\beta_{+}} \cdot x$, where $\mathfrak{r}^{\beta_{+}}$is the Lie algebra of $R^{\beta_{+}}$; 
(iii) $H_{x}\left(\eta_{\mathfrak{p}}\right) \geqslant 0$ on $T_{x}\left(S_{\beta}\right)=\mathfrak{g} \cdot x+T_{x}\left(S^{\beta_{+}}\right)=\mathfrak{k} \cdot x+T_{x}\left(S^{\beta_{+}}\right)$;

(iv) $H_{x}\left(\eta_{\mathfrak{p}}\right)<0$ on $T_{x}\left(S_{\beta}\right)^{\perp}=(\mathfrak{g} \cdot x)^{\perp} \cap T_{x}\left(S^{\beta_{+}}\right)^{\perp}=(\mathfrak{k} \cdot x)^{\perp} \cap T_{x}\left(S^{\beta_{+}}\right)^{\perp}$.

Proof. The $K$-invariance of $\eta_{\mathfrak{p}}$ implies assertion (i). The other assertions follow from $(\mathfrak{g} \cdot x)^{\perp} \subset$ $(\mathfrak{p} \cdot x)^{\perp}=\operatorname{ker} d \mu_{\mathfrak{p}}(x)$, Proposition 2.5, Proposition 4.3(iii) and the fact that $d \beta_{X}(x): T_{x} X \rightarrow T_{x} X$ has strictly positive eigenvalues on $\mathfrak{r}^{\beta_{+}} \cdot x$.

Remark 6.7. We have a tangent space decomposition $T_{x}(G \cdot x)=T_{x}(K \cdot x) \oplus \mathfrak{p}^{\beta} \cdot x \oplus \mathfrak{r}^{\beta_{+}} \cdot x$. This follows from the decompositions $G=K \cdot G^{\beta_{+}}, G^{\beta_{+}}=G^{\beta} \cdot R^{\beta_{+}}$, the identity $K \cap G^{\beta_{+}}=K^{\beta}$ and the fact that $G^{\beta}$ acts on $X^{\beta}$ whereas $R^{\beta_{+}}$acts on the fibers of $p^{\beta_{+}}$(see $\S 4$ ). Thus, the behavior of $H_{x}\left(\eta_{\mathfrak{p}}\right)$ on $T_{x}(G \cdot x)$ is precisely described by Proposition 6.6(i) and (ii).

Corollary 6.8. If $\beta \in \mathcal{B}_{\mathfrak{p}}$ is such that $\|\beta\|^{2}$ is a minimum value of $2 \eta_{\mathfrak{p}}$, then the corresponding pre-stratum $S_{\beta}$ is open in $X$ and coincides with $\mathcal{S}_{G}\left(\mathcal{M}_{\mathfrak{p}}(\beta)\right)$.

Proof. Let $x \in \mathcal{C}_{\mathfrak{p}}$ such that $\mu_{\mathfrak{p}}(x)=\beta$. By Proposition 6.6, $S_{\beta}$ is open in $X$. It contains $\mathcal{M}_{\mathfrak{p}^{\beta}}(\beta) \cap$ $X^{\beta}=\mathcal{M}_{\mathfrak{p}}(\beta) \cap X^{\beta}=\mathcal{M}_{\mathfrak{p}}(\beta)$ since $\|\beta\|^{2}$ is a minimum. However, $\mathcal{S}_{G}\left(\mathcal{M}_{\mathfrak{p}}(\beta)\right)$ is the smallest $G$-stable open set containing $\mathcal{M}_{\mathfrak{p}}(\beta)$, so that $\mathcal{S}_{G}\left(\mathcal{M}_{\mathfrak{p}}(\beta)\right) \subset S_{\beta}$. On the other hand,

$$
S_{\beta}=G \cdot S_{G^{\beta}}\left(\mathcal{M}_{\mathfrak{p}^{\beta}}(\beta) \cap X^{\beta}\right)\left(X^{\beta_{+}}\right) \subset S_{G}\left(\mathcal{M}_{\mathfrak{p}}(\beta)\right) .
$$

The argument in the proof shows the following more general result.

Corollary 6.9. Let $x$ be a local minimum of $\eta_{\mathfrak{p}}$. Then $x \in \mathcal{C}_{\mathfrak{p}}$ and the union of the connected components of the corresponding stratum $S_{\beta}$ which intersect $K \cdot x$ non-trivially is an open subset of $X$.

Corollary 6.10. Suppose that $X=G \cdot x$ where $x \in \mathcal{C}_{\mathfrak{p}}$ and $\beta:=\mu_{\mathfrak{p}}(x)$. Then:

(i) $G^{\beta_{+}} \cdot x=S^{\beta_{+}}$;

(ii) $\mathcal{S}_{G^{\beta}}\left(\mathcal{M}_{\mathfrak{p}^{\beta}}(\beta)\right)\left(X^{\beta}\right)=G^{\beta} \cdot x$;

(iii) $K^{\beta} \cdot x=\mathcal{M}_{\mathfrak{p}}(\beta)$;

(iv) $\mathcal{C}_{\mathfrak{p}}=K \cdot x$.

Proof. In the homogeneous case Theorem 5.3 (the slice theorem for pre-strata) gives us a $G$ equivariant isomorphism $G \times{ }^{G^{\beta_{+}}} S^{\beta_{+}} \rightarrow G \cdot x$ and therefore a $G$-equivariant map $p: G \cdot x \rightarrow G / G^{\beta_{+}}$ with $G^{\beta_{+}} \cdot x=p^{-1}\left(G^{\beta_{+}}\right)=S^{\beta_{+}}$. This shows part (i).

Let $z=q \cdot x \in S^{\beta_{+}}$where $q \in G^{\beta_{+}}$. Then $\lim _{t \rightarrow-\infty} \exp (t \beta) \cdot q \cdot x=h \cdot x$ where $h=$ $\lim _{t \rightarrow-\infty} \exp (t \beta) q \exp (-t \beta) \in G^{\beta}$. Hence, we have part (ii).

From part (ii) we obtain that $\mathcal{M}_{\mathfrak{p}^{\beta}}(\beta) \cap X^{\beta}=K^{\beta} \cdot x$. This implies part (iii). Now part (iii) gives $\mathcal{M}_{\mathfrak{p}}(K \cdot \beta)=K \cdot x$ and part (iv) follows from Lemma 6.1 .

Corollary 6.11. Let $x \in \mathcal{C}_{\mathfrak{p}}$. Then $x$ is a global minimum of $\left.\eta_{\mathfrak{p}}\right|_{G \cdot x}$.

Corollary 6.12. If $\eta_{\mathfrak{p}} \mid G \cdot x$ has a local maximum at $x$, then $G \cdot x=K \cdot x$.

Proof. If $x$ is a local maximum of $\eta_{\mathfrak{p}}$, then it is a critical point of $\eta_{\mathfrak{p}}$. However, every critical point of $\eta_{\mathfrak{p}}$ on $G \cdot x$ is a global minimum. This implies that the set of critical points, which is a $K$-orbit, is open in $G \cdot x$. Since $K$ intersects every component of $G$, we have $K \cdot x=G \cdot x$.

Corollary 6.13. If a $G$-orbit $G \cdot x$ in $X$ is compact, then $G \cdot x=K \cdot x$.

In Corollary 6.12 we have a special case of the existence of a maximal pre-stratum $S_{\beta}$. By this we mean that $\|\beta\| \geqslant\left\|\mu_{\mathfrak{p}}(z)\right\|$ holds for every $z \in X$. In this case $\eta_{\mathfrak{p}} \mid S_{\beta}$ is constant and $\mu_{\mathfrak{p}}$ 


\section{STRATIFiCATions}

maps $S_{\beta}$ equivariantly onto $K \cdot \beta$. This implies that every $G$-orbit in $S_{\beta}$ is a $K$-orbit, hence closed. More precisely, we have $S_{\beta}=\mathcal{M}_{\mathfrak{p}}(K \cdot \beta) \cap S_{\beta}$ and $S^{\beta_{+}}=\mathcal{M}_{\mathfrak{p}^{\beta}}(\beta) \cap S^{\beta_{+}}=\mathcal{M}_{\mathfrak{p}}(\beta) \cap X^{\beta}$. Note that this implies that $S^{\beta_{+}}$coincides with a union of connected components of $X^{\beta}$. Also since $S_{\beta} \cong K \times{ }^{K^{\beta}}\left(\mathcal{M}_{\mathfrak{p}^{\beta}}(\beta) \cap X^{\beta}\right)$ we have that $S_{\beta}$ is compact if $X^{\beta}$ is compact or $\mu_{\mathfrak{p}}$ is a proper map.

As an application, we show how our results imply Matsuki duality.

Corollary 6.14. Assume that $X=Z$ is $U$-homogeneous, i.e. a generalized flag manifold, and assume that $G$ is a real form of $U^{\mathbb{C}}$. Then each $G$-orbit and each $K^{\mathbb{C}}$-orbit intersect $\mathcal{C}_{\mathfrak{p}}$ in a unique $K$-orbit (Matsuki duality). If $x \in \mathcal{C}_{\mathfrak{p}}$, then $K \cdot x$ is the set of global minima of $\eta_{\mathfrak{p}}$ on $G \cdot x$ and of $\eta_{\mathfrak{k}}$ on $K^{\mathbb{C}} \cdot x$. Thus, $G \cdot x \cap K^{\mathbb{C}} \cdot x=K \cdot x$. Moreover, $K^{\mathbb{C}} \cdot x$ (respectively $G \cdot x$ ) is open in $X$ if and only if $G \cdot x=K \cdot x$ (respectively $K^{\mathbb{C}} \cdot x=K \cdot x$ ).

Proof. We have $T_{x} X=T_{x}(G \cdot x)+T_{x}\left(K^{\mathbb{C}} \cdot x\right)$. We may assume that the decomposition $\mathfrak{u}^{\mathbb{C}}=$ $\mathfrak{k} \oplus \mathfrak{i k} \oplus \mathfrak{i p} \oplus \mathfrak{p}$ is orthogonal with respect to a $U$-invariant inner product on $\mathfrak{u}^{\mathbb{C}}$. Then $\frac{1}{2}\|\mu\|^{2}=\eta_{\mathfrak{k}}+\eta_{\mathfrak{p}}$ where $\eta_{\mathfrak{k}}:=\frac{1}{2}\left\|\mu_{\mathfrak{k}}\right\|^{2}$. Since $Z$ is $U$-homogeneous this function is constant and $\eta_{\mathfrak{k}}$ is the negative of $\eta_{\mathfrak{p}}$ up to a constant. In particular, the set of critical points of $\eta_{\mathfrak{k}}$ equals $\mathcal{C}_{\mathfrak{p}}$. Using the behavior of the Hessian of $\eta_{\mathfrak{p}}$ (respectively $\eta_{\mathfrak{k}}$ ) described in Proposition 6.6, we get that each $G$-orbit (respectively $K^{\mathbb{C}}$-orbit) is open in the $G$-pre-stratum (respectively $K^{\mathbb{C}}$-pre-stratum) in which it is contained. Therefore, each $G$-orbit and each $K^{\mathbb{C}}$-orbit intersects $\mathcal{C}_{\mathfrak{p}}$ in a unique $K$-orbit. If $x \in \mathcal{C}_{\mathfrak{p}}$, then $K \cdot x$ is the set of global minima of $\eta_{\mathfrak{p}}$ on $G \cdot x$ and of $\eta_{\mathfrak{k}}$ on $K^{\mathbb{C}} \cdot x$. Again, since $\frac{1}{2}\|\mu\|^{2}$ is constant, we have $G \cdot x \cap K^{\mathbb{C}} \cdot x=K \cdot x$. The last statement follows from the identities $G \cdot x \cap K^{\mathbb{C}} \cdot x=K \cdot x$ and $T_{x} X=T_{x}(G \cdot x)+T_{x}\left(K^{\mathbb{C}} \cdot x\right)$.

Remark 6.15. Matsuki duality does not depend on the choice of the moment map, as follows. Assume that we are given another $U$-equivariant moment map $\tilde{\mu}: Z \rightarrow \mathfrak{u}^{*}$. Let $\tilde{\eta}_{\mathfrak{p}}:=\frac{1}{2}\left\|\tilde{\mu}_{\mathfrak{p}}\right\|^{2}$ and let $\tilde{\mathcal{C}}_{\mathfrak{p}}$ denote the set of critical points of $\tilde{\eta}_{\mathfrak{p}}$. If there is an $x_{0} \in \mathcal{C}_{\mathfrak{p}}$ where $x_{0} \notin \tilde{\mathcal{C}}_{\mathfrak{p}}$, then, by Matsuki duality, there is an $x_{1} \in K^{\mathbb{C}} \cdot x_{0} \cap \tilde{\mathcal{C}}_{\mathfrak{p}}$ with $x_{1} \notin \mathcal{C}_{\mathfrak{p}}$ and $\eta_{\mathfrak{p}}\left(x_{0}\right)>\eta_{\mathfrak{p}}\left(x_{1}\right)$. Again, by Matsuki duality, we obtain an $x_{2} \in G \cdot x_{1} \cap \mathcal{C}_{\mathfrak{p}}$ with $x_{2} \notin \tilde{\mathcal{C}}_{\mathfrak{p}}$ and $\eta_{\mathfrak{p}}\left(x_{1}\right)>\eta_{\mathfrak{p}}\left(x_{2}\right)$. Inductively we obtain a sequence $x_{n}$ in $\mathcal{C}_{\mathfrak{p}}$ with $\eta_{\mathfrak{p}}\left(x_{n}\right)>\eta_{\mathfrak{p}}\left(x_{n+1}\right)$. However, $\mathcal{C}_{\mathfrak{p}}$ consists of finitely many $K$-orbits so $K \cdot x_{n_{0}}=K \cdot x_{0}$ for some $n_{0}>0$, which contradicts the inequality $\eta_{\mathfrak{p}}\left(x_{0}\right)>\eta_{\mathfrak{p}}\left(x_{n_{0}}\right)$. Hence, $\tilde{\mathcal{C}}_{\mathfrak{p}}=\mathcal{C}_{\mathfrak{p}}$.

We can also easily obtain an old result of Wolf [Wol69].

Corollary 6.16. Assume that $X=Z$ is $U$-homogeneous and that $G$ is a real form of $U^{\mathbb{C}}$. Then there is exactly one closed $G$-orbit in $X$.

Proof. If $G \cdot x$ is a closed orbit in $Z$ it equals $K \cdot x$ by Corollary 6.13 . Then we may assume $x \in \mathcal{C}_{\mathfrak{p}}$. By Corollary 6.14 the dual orbit $K^{\mathbb{C}} \cdot x$ is open. Since $K^{\mathbb{C}}$ is complex and acts holomorphically on $Z$ there is only one open orbit.

\section{Proper moment maps}

In this section we consider what happens when $\mu_{\mathfrak{p}}: X \rightarrow \mathfrak{p}$ is a proper map. In this case $\eta_{\mathfrak{p}}: X \rightarrow \mathbb{R}$ is a proper non-negative function, i.e. an exhaustion of $X$. Note that properness of $\eta_{\mathfrak{p}}$ implies that $X$ is a closed $G$-stable submanifold of $Z$.

Let $(t, x) \mapsto \varphi_{t}(x)$ denote the local flow of the vector field $\operatorname{grad} \eta_{\mathfrak{p}}$ on $X$. Properness of $\mu_{\mathfrak{p}}$ implies that $\varphi_{t}$ exists for all negative $t$. By Lemma 2.3 , the flow $\varphi_{t}$ is along $G$-orbits. Note that $\eta_{\mathfrak{p}}\left(\varphi_{t}(x)\right)$ is strictly increasing unless $x$ is a critical point of $\eta_{\mathfrak{p}}$. For $z \in X$ let $\mathcal{L}(z)$ denote the set of points $y \in X$ such that $y=\lim _{k \rightarrow \infty} \varphi_{t_{k}}(z)$ for some sequence $t_{k}$ which goes to $-\infty$. Since we assume that $\mu_{\mathfrak{p}}$ is proper we have the following. 


\section{P. Heinzner, G. W. Schwarz and H. Stötzel}

Lemma 7.1. Let $z \in X$. Then $\mathcal{L}(z)$ is non-empty and for every open neighborhood $V$ of $\mathcal{L}(z)$ there exists a $t_{0} \in \mathbb{R}$ such that $\varphi_{t}(z) \in V$ for every $t<t_{0}$.

Proof. Let $I_{V}:=\left\{t \in \mathbb{R}: t \leqslant 0\right.$ and $\left.\varphi_{t}(z) \in X \backslash V\right\}$. Since $V$ contains $\mathcal{L}(z)$ the properness of $\eta_{\mathfrak{p}}$ implies that $I_{V}$ is compact. For $t_{0}:=\min \left\{t \in I_{V}\right\}$ we have $\varphi_{t}(z) \in V$ for all $t<t_{0}$.

For $\beta \in \mathcal{B}_{\mathfrak{p}}$ let $\mathcal{C}_{\mathfrak{p}}(K \cdot \beta)$ denote $\mathcal{C}_{\mathfrak{p}} \cap \mathcal{M}_{\mathfrak{p}}(K \cdot \beta)$ and set $\mathcal{C}_{\mathfrak{p}}(\|\beta\|)=\mathcal{C}_{\mathfrak{p}} \cap \eta_{\mathfrak{p}}^{-1}\left(\frac{1}{2}\|\beta\|^{2}\right)$. If we set $\beta:=\mu_{\mathfrak{p}}(x)$ for some $x \in \mathcal{L}(z)$, then $x \in \mathcal{C}_{\mathfrak{p}}(K \cdot \beta)=\mathcal{C}_{\mathfrak{p}}(K \cdot \beta) \cap S_{\beta} \subset \mathcal{M}_{\mathfrak{p}}(K \cdot \beta) \cap S_{\beta}$. It is a consequence of Lemma 7.1 that $\mathcal{L}(z)$ is connected. Then $\eta_{\mathfrak{p}}$ is constant on $\mathcal{L}(z)$ and we have

$$
\mathcal{L}(z) \subset \bigcup_{\|\tilde{\beta}\|=\|\beta\|} \mathcal{C}_{\mathfrak{p}}(K \cdot \tilde{\beta})=\mathcal{C}_{\mathfrak{p}}(\|\beta\|) .
$$

We need the following technical result whose proof we give at the end of this section.

Proposition 7.2. Let $z \in X$ and choose $\beta \in \mathcal{B}_{\mathfrak{p}}$ such that $\mathcal{L}(z) \cap \mathcal{C}_{\mathfrak{p}}(K \cdot \beta) \neq \emptyset$. Then there is an open neighborhood $\Omega$ of $\mathcal{C}_{\mathfrak{p}}(K \cdot \beta)$ and a smooth function $\rho: \Omega \rightarrow \mathbb{R}$ such that:

(i) $\rho \geqslant 0$;

(ii) $\Omega \cap S_{\beta}=\{z \in \Omega: \rho(z)=0\}$;

(iii) $d \rho\left(\operatorname{grad}\left(\eta_{\mathfrak{p}}\right)\right)(z) \leqslant 0$ for all $z \in \Omega$.

The proposition has the following consequence for the flow $\varphi_{t}$.

Theorem 7.3 (Stratification Theorem). If $\mu_{\mathfrak{p}}: X \rightarrow \mathfrak{p}$ is proper, then $X=\bigcup_{\beta} S_{\beta}$ where $\beta$ runs through a complete set of representatives of $K$-orbits in $\mathcal{B}_{\mathfrak{p}}$. The union is disjoint, each $S_{\beta}$ is a locally closed submanifold of $X$ and

$$
\overline{S_{\beta}} \subset S_{\beta} \cup\left\{S_{\gamma}:\|\gamma\|>\|\beta\|\right\} .
$$

Proof. Let $z \in X$ and $\beta \in \mathcal{B}_{\mathfrak{p}}$ such that $\mathcal{L}(z) \cap \mathcal{C}_{\mathfrak{p}}(K \cdot \beta) \neq \emptyset$. Choose an open neighborhood $\Omega$ of $\mathcal{C}_{\mathfrak{p}}(K \cdot \beta)$ which has the properties given in Proposition 7.2.

Let $\Omega_{0}$ be an open neighborhood of $\mathcal{C}_{\mathfrak{p}}(K \cdot \beta)$ which is relatively compact in $\Omega$ and let $Q:=$ $\Omega_{0} \cap \mathcal{C}_{\mathfrak{p}}(\|\beta\|)$. Since $\mathcal{C}_{\mathfrak{p}}(\|\beta\|) \backslash Q$ is compact and $\left(\mathcal{C}_{\mathfrak{p}}(\|\beta\|) \backslash Q\right) \cap \overline{S_{\beta}}=\emptyset$ there is an open relatively compact neighborhood $V^{\prime}$ of $\mathcal{C}_{\mathfrak{p}}(\|\beta\|) \backslash Q$ in $X$ such that $\overline{V^{\prime}} \cap \overline{S_{\beta}}=\emptyset$. Then the set $V:=V^{\prime} \cup \Omega_{0}$ is an open neighborhood of $\mathcal{L}(z) \subset \mathcal{C}_{\mathfrak{p}}(\|\beta\|)$. Let $r:=\min \left\{\rho(y): y \in \overline{V^{\prime}} \cap \overline{\Omega_{0}}\right\}$. Since $\overline{V^{\prime}} \cap S_{\beta}=\emptyset$ we have $\rho(y)>0$ on the compact set $\overline{V^{\prime}} \cap \overline{\Omega_{0}}$ and therefore $r>0$. The set $\Omega_{1}:=\left\{y \in \Omega_{0}: \rho(y)<r\right\}$ is an open neighborhood of $\mathcal{C}_{\mathfrak{p}}(K \cdot \beta)$ such that $\overline{\Omega_{1}} \cap V \subset \Omega$.

Since $\mathcal{L}(z) \cap \mathcal{C}_{\mathfrak{p}}(K \cdot \beta) \neq \emptyset$ there exists a $t_{0}$ with $\varphi_{t_{0}}(z) \in \Omega_{1}$. By Lemma 7.1 we may assume that $\varphi_{t}(z) \in V$ for all $t \leqslant t_{0}$. We claim that $\varphi_{t}(z) \in \Omega_{1}$ for all $t \leqslant t_{0}$. It suffices to show that $I_{\Omega_{1}}:=\left\{t \in \mathbb{R}: t \leqslant t_{0}, \varphi_{t}(z) \in \Omega_{1}\right\}$ is connected since it is not bounded from below. If this would not be the case then we would find a connected component $I_{1}=\left(a_{1}, t_{1}\right)=\left\{t \in I_{\Omega_{1}}: a_{1}<t<t_{1}\right\}$ of $I_{\Omega_{1}}$ where $a_{1}$ is possibly $-\infty$ and $t_{1}<t_{0}$. Then $\varphi_{t_{1}}(z) \in V \cap\left(\overline{\Omega_{1}} \backslash \Omega_{1}\right)$, i.e. $\rho\left(\varphi_{t_{1}}(z)\right)=r$, and $\rho\left(\varphi_{t}(z)\right)<r$ for all $t \in I_{1}$. This contradicts $a_{1}<t_{1}<t_{0}$, since $t \mapsto \rho\left(\varphi_{-t}(z)\right)$ is defined and increasing for all $t$ in a sufficiently small open interval which contains $t_{1}$.

Now assume that $z \notin S_{\beta}$. Since $\rho\left(\varphi_{t}(z)\right)>0$ for all $t<t_{0}$ and $t \mapsto \rho\left(\varphi_{-t}(z)\right)$ is increasing there is no $x \in \mathcal{L}(z) \cap \mathcal{C}_{\mathfrak{p}}(K \cdot \beta) \subset S_{\beta}$ with $\rho(x)=0$. This contradiction proves that $z \in S_{\beta}$ and shows that $X=\bigcup_{\beta} S_{\beta}$. The union is disjoint and we also have $\overline{S_{\beta}} \subset S_{\beta} \cup\left\{S_{\gamma}:\|\gamma\|>\|\beta\|\right\}$ by Theorem 5.5 (the pre-stratification theorem).

In the proof we have seen that $z \in S_{\beta}$ if $\mathcal{L}(z) \cap \mathcal{C}_{\mathfrak{p}}(K \cdot \beta) \neq \emptyset$. Since $\mathcal{L}(z) \subset \mathcal{C}_{\mathfrak{p}}(\|\beta\|)$ we obtain the following. 


\section{STRATIFICATIONS}

Corollary 7.4. Let $z \in S_{\beta}$. Then $\mathcal{L}(z) \subset \mathcal{C}_{\mathfrak{p}}(K \cdot \beta)$.

Remark 7.5. The map $\varphi:(-\infty, 0] \times S_{\beta} \rightarrow S_{\beta},(t, x) \mapsto \varphi_{t}(x)$ extends to a continuous map $\bar{\varphi}:[-\infty, 0] \times S_{\beta} \rightarrow S_{\beta}$. Then $\bar{\varphi}$ is a $K$-equivariant strong deformation retraction of $S_{\beta}$ onto $\mathcal{C}_{\mathfrak{p}}(K \cdot \beta)$ which stabilizes closures of $G$-orbits. In particular, the set $\mathcal{L}(z)$ consists of one point. For the proof one uses a result of Marle [Mar84] and Guillemin and Sternberg [GS84] which states that there exist local coordinates at any $z \in Z$ in which the moment map $\mu$ is real analytic. Applying this to points $x \in \mathcal{C}_{\mathfrak{p}}(K \cdot \beta)$ one obtains the existence of $\bar{\varphi}$ as in [Nee85] (see [Sch89]) using an inequality of Łojasiewicz.

If $\mu_{\mathfrak{p}}$ is not assumed to be proper, one can prove that $\bar{\varphi}$ realizes $\mathcal{M}_{\mathfrak{p}}$ as a strong deformation retract of a neighborhood of $\mathcal{M}_{\mathfrak{p}}$ in $S_{0}=\mathcal{S}_{G}\left(\mathcal{M}_{\mathfrak{p}}\right)$. Here the same method applies since in [HS07b] it is shown that there exist relatively compact neighborhoods of points in $\mathcal{M}_{\mathfrak{p}}$ which have the property that they contain $\varphi_{t}(z)$ for all $t<t_{0}$ if they contain $\varphi_{t_{0}}(z)$.

We have the following characterization of $S_{\beta}$.

Corollary 7.6. Let $\mu_{\mathfrak{p}}$ be proper and $\beta \in \mathcal{B}_{\mathfrak{p}}$. Then

$$
\begin{aligned}
S_{\beta} & =\left\{z \in X: \beta \in \mu_{\mathfrak{p}}(\overline{G \cdot z}) \text { and }\|\beta\| \leqslant\left\|\mu_{\mathfrak{p}}(g \cdot z)\right\| \text { for all } g \in G\right\} \\
& =\left\{z \in X: \lim _{t \rightarrow-\infty} \varphi_{t}(z) \in \mathcal{C}_{\mathfrak{p}}(K \cdot \beta)\right\} .
\end{aligned}
$$

Remark 7.7. In general we have $S_{\beta} \subset\left\{z \in X: \beta \in \mu_{\mathfrak{p}}(\overline{G \cdot z})\right.$ and $\|\beta\| \leqslant\left\|\mu_{\mathfrak{p}}(g \cdot z)\right\|$ for all $\left.g \in G\right\}$.

We now work towards a proof of Proposition 7.2.

As in $\S 2$, if $x$ is a zero of a vector field $\zeta$ on $X$, then $d \zeta(x)$ denotes the corresponding endomorphism of $T_{x}(X)$.

Lemma 7.8. For $x \in \mathcal{C}_{\mathfrak{p}}$ and $\beta:=\mu_{\mathfrak{p}}(x)$ we have:

(i) $d \operatorname{grad} \eta_{\mathfrak{p}}(x) \cdot v=\left(d \beta_{X}(x)\right) \cdot v+\left(d \mu_{\mathfrak{p}}(x) \cdot v\right)_{X}(x)$ for all $v \in T_{x}(X)$; and

(ii) the linear map $L: T_{x}(X) \rightarrow T_{x}(X), v \mapsto\left(d \mu_{\mathfrak{p}}(x) \cdot v\right)_{X}(x)$ maps $T_{x}\left(S_{\beta}\right)$ into itself and is zero on $T_{x}\left(S_{\beta}\right)^{\perp}$.

Proof. Let $\gamma$ be a smooth curve through $x \in \mathcal{C}_{\mathfrak{p}}$ such that $\gamma(0)=x$ and set

$$
v:=\left.\frac{d}{d t}\right|_{0} \gamma(t)
$$

We have $\operatorname{grad} \eta_{\mathfrak{p}}(\gamma(t))=\mu_{\mathfrak{p}}(\gamma(t))_{X}(\gamma(t))=\operatorname{grad} \mu_{\mathfrak{p}}^{\mu_{\mathfrak{p}}(\gamma(t))}(\gamma(t))$ and $\mu_{\mathfrak{p}}(\gamma(t))=\mu_{\mathfrak{p}}(x)+t \cdot d \mu_{\mathfrak{p}}(x) \cdot v+$ $t^{2} R(t)$ for a continuous map $R(t)$. Since $\operatorname{grad} \eta_{\mathfrak{p}}(\gamma(t))=\operatorname{grad} \mu_{\mathfrak{p}}^{\mu_{\mathfrak{p}}(x)}(\gamma(t))+t \operatorname{grad} \mu_{\mathfrak{p}}^{d \mu_{\mathfrak{p}}(x) \cdot v}(\gamma(t))+$ $t^{2} \operatorname{grad} \mu_{\mathfrak{p}}^{R(t)}(\gamma(t))$ we have $d \operatorname{grad} \eta_{\mathfrak{p}}(x) \cdot v=d \beta_{X}(x) \cdot v+\left(d \mu_{\mathfrak{p}}(x) \cdot v\right)_{X}(x)$ proving part (i). We also have part (ii), since $\mathfrak{g} \cdot x \subset T_{x}\left(S_{\beta}\right)$ and $\operatorname{ker} d \mu_{\mathfrak{p}}(x)=(\mathfrak{p} \cdot x)^{\perp}$.

Proof of Proposition 7.2. Let $\Omega_{0}$ be an open neighborhood of $S_{\beta}$ in $X$ which is diffeomorphic to the normal bundle $T\left(S_{\beta}\right)^{\perp} \mid S_{\beta}$ of $S_{\beta}$ in $X$ such that $S_{\beta}$ corresponds to the zero section. Using this identification we define $\rho: \Omega_{0} \rightarrow \mathbb{R}, \rho(v)=(v, v)_{\pi(v)}$ where $\pi: T\left(S_{\beta}\right)^{\perp} \mid S_{\beta} \rightarrow S_{\beta}$ denotes the canonical projection and $(\cdot, \cdot)_{\pi(v)}$ denotes the inner product on $T_{\pi(v)}(X)$. Then we have $\rho \geqslant 0$ and $\Omega_{0} \cap S_{\beta}=\left\{y \in \Omega_{0}: \rho(y)=0\right\}$.

For $x_{0} \in \mathcal{C}_{\mathfrak{p}}(K \cdot \beta)$ there exists an open neighborhood $U_{0}$ of $x_{0}$ in $\Omega_{0}$ which can be identified with $\mathbb{R}^{n} \times \mathbb{R}^{m}$ where $x_{0}$ corresponds to $0, S_{\beta}$ corresponds to $\mathbb{R}^{n} \times\{0\}$ and the normal space $T_{x}\left(S_{\beta}\right)^{\perp}$ corresponds to $\{x\} \times \mathbb{R}^{m}$ for $x \in U_{0} \cap S_{\beta}$. Furthermore, this identification can be chosen such that $\rho$ has the form $\rho\left(v_{1}, v_{2}\right)=\left\|v_{2}\right\|^{2}$ on $\mathbb{R}^{n} \times \mathbb{R}^{m}$. 


\section{P. Heinzner, G. W. Schwarz and H. Stötzel}

Expressing the content of Lemma 7.8 in these coordinates we obtain

$$
\operatorname{grad} \eta_{\mathfrak{p}}\left(v_{1}, v_{2}\right)=d \beta_{X}(0)\left(v_{1}, v_{2}\right)+L\left(v_{1}\right)+\mathrm{O}\left(\left\|v_{1}\right\|^{2}+\left\|v_{2}\right\|^{2}\right)
$$

where $L$ is a linear mapping from $\mathbb{R}^{n}$ to $\mathbb{R}^{n}$.

Since $d \beta_{X}(0)$ has only negative eigenvalues on $\mathbb{R}^{m}$, the quadratic form $v_{2} \mapsto\left(v_{2}, d \beta_{X}(0) \cdot v_{2}\right)$ is strictly negative definite. Furthermore, $d \beta_{X}(0)$ preserves $T_{0}\left(S_{\beta}\right)$ and $T_{0}\left(S_{\beta}\right)^{\perp}$, so we have

$$
d \rho\left(\operatorname{grad} \eta_{\mathfrak{p}}\right)\left(v_{1}, v_{2}\right)=\left(v_{2}, d \beta_{X}(0) \cdot v_{2}\right)+\mathrm{O}\left(\left\|v_{1}\right\|^{3}+\left\|v_{2}\right\|^{3}\right) .
$$

Thus, after possibly shrinking $U_{0}$ we can find a $c<0$ such that $d \rho\left(\operatorname{grad} \eta_{\mathfrak{p}}\right)\left(v_{1}, v_{2}\right) \leqslant c \cdot \rho\left(v_{1}, v_{2}\right)$ for $\left(v_{1}, v_{2}\right) \in U_{0}$.

Since $x_{0} \in \mathcal{C}_{\mathfrak{p}}(K \cdot \beta)$ was arbitrary we find a neighborhood $\Omega \subset \Omega_{0}$ of $\mathcal{C}_{\mathfrak{p}}(K \cdot \beta)$ in $X$ such that $d \rho\left(\operatorname{grad} \eta_{\mathfrak{p}}\right)(z) \leqslant 0$ if $z \in \Omega$.

\section{Compact manifolds}

In this section we show that for a compact $G$-stable submanifold $X$ of $Z$ there are only finitely many pre-strata. Let $\mathfrak{a}$ be a maximal subalgebra of $\mathfrak{p}$ and $A=\exp \mathfrak{a}$ the corresponding subgroup of $G$. Note that $A$ is compatible with the Cartan decomposition of $U^{\mathbb{C}}$. Since the corresponding $A$ gradient map $\mu_{\mathfrak{a}}$ is locally constant on the smooth compact manifold $X^{A}$ we find that $\mathcal{V}:=\mu_{\mathfrak{a}}\left(X^{A}\right)$ consists of finitely many points.

In [HS07b] the following is established.

Lemma 8.1. For $x \in X$ the image $\mu_{\mathfrak{a}}(A \cdot x)$ is an open convex subset of the affine subspace $\mu_{\mathfrak{a}}(x)+\mathfrak{a}_{x}^{\perp}$ of $\mathfrak{a}$ where $\mathfrak{a}_{x}^{\perp}:=\left\{\beta \in \mathfrak{a} ;\langle\alpha, \beta\rangle=0\right.$ for all $\left.\alpha \in \mathfrak{a}_{x}\right\}$ denotes the orthogonal complement of $\mathfrak{a}_{x}$ in $\mathfrak{a}$.

Proposition 8.2. Let $C \subset X$ be compact and $A$-invariant. If the image $\mu_{\mathfrak{a}}(C)$ is convex, then it is the convex hull of a subset of $\mathcal{V}$. In particular, it is a polytope.

Proof. Since $\mu_{\mathfrak{a}}(C)$ is compact and convex it is the convex hull of the set of its extreme points. Let $\beta$ be an extreme point and let $x \in C$ with $\mu_{\mathfrak{a}}(x)=\beta$. Since $C$ contains $A \cdot x$, Lemma 8.1 implies that $\beta$ lies in an open subset of $\beta+\mathfrak{a}_{x}^{\perp}$ which is contained in $\mu_{\mathfrak{a}}(C)$. However, $\beta$ is an extreme point so $\mathfrak{a}_{x}^{\perp}=\{0\}$. Therefore, $\mathfrak{a}_{x}=\mathfrak{a}$ and consequently $x \in X^{A}$.

Corollary 8.3. Let $X$ be compact and $x \in X$. Then the image $\mu_{\mathfrak{a}}(\overline{A \cdot x})$ is the convex hull of a subset of $\mathcal{V}$.

Corollary 8.4. Let $X$ be compact and $\beta \in \mathcal{B}_{\mathfrak{p}} \cap \mathfrak{a}$. Then $\beta$ is the closest point to zero in the convex hull of finitely many elements of $\mathcal{V}$. In particular, $\mathcal{B}_{\mathfrak{p}} \cap \mathfrak{a}$ is a finite set and $\mathcal{B}_{\mathfrak{p}}$ consists of finitely many $K$-orbits.

Proof. Let $x \in \mathcal{C}_{\mathfrak{p}}$ such that $\mu_{\mathfrak{p}}(x)=\beta$. Note that $x$ is also a critical point for $\eta_{\mathfrak{a}}$. Applying Proposition 5.13 to the group $A$ and $Y_{\beta}:=\overline{A \cdot x}$, we obtain that $\|\beta\| \leqslant\|\zeta\|$ for all $\zeta \in \mu_{\mathfrak{a}}\left(Y_{\beta}\right)$. Since $\mu_{\mathfrak{a}}\left(Y_{\beta}\right)$ is the convex hull of images of $A$-fixed points, $\beta$ is the closest point to the origin of a subset of $\mathcal{V}$.

Corollary 8.5. If $X$ is compact, then there are only finitely many pre-strata.

In the case where $X$ is compact we call a pre-stratum $S_{\beta}$ a stratum.

\section{Morse inequalities}

Let $G$ be an arbitrary Lie group and $Y$ a topological $G$-space. The $G$-equivariant cohomology $H_{G}^{n}(Y)$ of $Y$ is by definition the ordinary cohomology $H^{n}\left(\mathrm{EG} \times{ }^{G} Y\right)$ where EG is the total space of 


\section{StRATificATIONS}

a universal $G$-bundle $p: \mathrm{EG} \rightarrow \mathrm{BG}$ and $\mathrm{EG} \times{ }^{G} Y$ is the quotient of $\mathrm{EG} \times Y$ by the diagonal action of $G$.

We define the $G$-equivariant Poincaré series to be the power series $P_{t}^{G}(Y):=\sum_{n=0}^{\infty} t^{n} \operatorname{dim} H_{G}^{n}(Y)$ where cohomology is computed with respect to a field $\mathbb{K}$ which we omit in the notation. If $G=\{e\}$ is the trivial group, equivariant cohomology coincides with ordinary cohomology and we write $P_{t}(Y)$ for $P_{t}^{G}(Y)$.

Now assume that $X$ is an orientable connected compact $G$-invariant submanifold of $Z$ and that $G$ is a closed connected compatible subgroup of $U^{\mathbb{C}}$. Consider the finite decomposition $X=\bigcup_{\beta} S_{\beta}$ (Theorem 7.3 and Corollary 8.4). If $S_{\beta, m}$ denotes the union of the connected components of $S_{\beta}$ which have codimension $m$ in $X$, then $X=\bigcup_{\beta, m} S_{\beta, m}$ is again a decomposition of $X$ with closures of strata having the properties analogous to those in Theorem 7.3. In the following, cohomology is computed with coefficients in $\mathbb{Q}$ if all of the strata $S_{\beta}$ are orientable submanifolds of $X$ and with coefficients in $\mathbb{Z}_{2}=\mathbb{Z} / 2$ otherwise.

Standard considerations in equivariant topology show that we have the following.

Morse inequalities. There exists a series $R(t)$ with non-negative integer coefficients such that

$$
\sum_{\beta, m} t^{m} P_{t}^{K}\left(S_{\beta, m}\right)-P_{t}^{K}(X)=(1+t) R(t)
$$

where $\beta$ runs through a complete set of representatives of $K$-orbits in $\mathcal{B}_{\mathfrak{p}}$.

Since $K$ is a strong deformation retract of $G$ and the strata are $G$-stable the Morse inequalities are also valid if one replaces $K$-equivariant cohomology by $G$-equivariant cohomology.

The following result of [Kir84a] is useful for computing equivariant cohomology.

Result (Kirwan [Kir84a]). Let $Z$ be compact and $K$ compact and connected. Then $P_{t}^{K}(Z)=$ $P_{t}(Z) \cdot P_{t}(\mathrm{BK})$ where $\mathrm{BK}$ is the base space of a universal $K$-bundle.

In [Kir84a] it is shown that in the case where $X:=Z$ is compact and $G=K^{\mathbb{C}}$ is complex reductive the Morse inequalities are equalities, i.e. $R(t)=0$. Furthermore, the strata $S_{\beta}$ are complex and therefore orientable in this case. In our general situation where $G$ is not complex reductive, there may exist non-orientable strata.

Example 9.1. Let $X:=Z:=\mathbb{P}_{2}(\mathbb{C})$ be equipped with the standard action of $\mathrm{SL}_{3}(\mathbb{C})$ and with the moment map induced by the Fubini-Study metric. Then for $G=\mathrm{SL}_{3}(\mathbb{R})$ there are two strata, namely $\mathbb{P}_{2}(\mathbb{R})$, which is not orientable, and its complement.

We end with an example which shows that, in our situation, the Morse inequalities are not necessarily equalities, even in the case that $X=Z$.

Example 9.2. Let $U^{\mathbb{C}}=\mathrm{SL}_{2}(\mathbb{C})$ act on $X:=Z:=\mathbb{P}_{1}(\mathbb{C})$ by $g \cdot[z]:=[g z]$. Then the moment map with respect to the Fubini-Study metric is given by

$$
\mu^{\xi}([z])=\frac{\langle\xi \cdot z, z\rangle}{\mathrm{i}\langle z, z\rangle}
$$

where $\langle\cdot, \cdot\rangle$ is the standard Hermitian product on $\mathbb{C}^{2}$. Let $G=\mathrm{SL}_{2}(\mathbb{R})$. Then $K=\mathrm{SO}_{2}(\mathbb{R}) \cong S^{1}$ and $\mathfrak{p}$ is the set of real symmetric matrices of trace zero. One can show that $\mathbb{P}_{1}(\mathbb{C})$ decomposes into two orientable strata, namely $\mathcal{S}_{G}\left(\mathcal{M}_{\mathfrak{p}}\right)=G \cdot[1, \mathrm{i}] \cup G \cdot[1,-\mathrm{i}]$ and $S:=G \cdot[1,0]=K \cdot[1,0] \cong S^{1}$.

For $S^{1}$, a universal bundle is given by $p: S^{\infty} \rightarrow \mathbb{P}_{\infty}(\mathbb{C})$ where $S^{\infty}:=\lim _{n \rightarrow \infty} S^{2 n+1}, \mathbb{P}_{\infty}(\mathbb{C}):=$ $\lim _{n \rightarrow \infty} \mathbb{P}_{n}(\mathbb{C})$ and $p$ is induced by the projections $S^{2 n+1} \rightarrow S^{2 n+1} / S^{1} \cong \mathbb{P}_{n}(\mathbb{C})$ where $S^{1}$ acts by multiplication on $S^{2 n+1} \subset \mathbb{C}^{n+1}$. 
We have

$$
P_{t}^{K}\left(\mathbb{P}_{1}(\mathbb{C})\right)=P_{t}\left(\mathbb{P}_{1}(\mathbb{C})\right) \cdot P_{t}(\mathrm{BK})=\left(1+t^{2}\right) \sum_{n \geqslant 0} t^{2 n}=1+2 \sum_{n \geqslant 1} t^{2 n}
$$

Since $[1, \mathrm{i}]$ (respectively $[1,-\mathrm{i}]$ ) can be realized $K$-equivariantly as a strong deformation retract of $G \cdot[1, \mathrm{i}]$ (respectively $G \cdot[1,-\mathrm{i}])$, we have

$$
\begin{aligned}
P_{t}^{K}\left(\mathcal{S}_{G}\left(\mathcal{M}_{\mathfrak{p}}\right)\right) & =P_{t}^{K}(G \cdot[1, \mathrm{i}])+P_{t}^{K}(G \cdot[1,-\mathrm{i}])=2 \cdot P_{t}\left(E K \times_{K}[1, \mathrm{i}]\right) \\
& =2 \cdot P_{t}(B K)=2 \cdot \sum_{n \geqslant 0} t^{2 n} .
\end{aligned}
$$

For the second stratum we get

$$
P_{t}^{K}(S)=P_{t}(\mathrm{EK})=1
$$

Finally we obtain the Morse inequalities

$$
\begin{aligned}
t^{0} \cdot P_{t}^{K}\left(\mathcal{S}_{G}\left(\mathcal{M}_{\mathfrak{i p}}\right)\right)+t^{1} \cdot P_{t}^{K}(S)-P_{t}^{K}\left(\mathbb{P}_{1}(\mathbb{C})\right) & =2 \sum_{n \geqslant 0} t^{2 n}+t-\left(1+2 \sum_{n \geqslant 1} t^{2 n}\right) \\
& =1+t .
\end{aligned}
$$

Thus, $R(t)=1 \neq 0$.

\section{ACKNOWLEDGEMENTS}

The authors thank the referee for helpful remarks.

\section{REFERENCES}

Ati82 M. F. Atiyah, Convexity and commuting Hamiltonians, Bull. London Math. Soc. 14 (1982), 1-15.

BL02 R. Bremigan and J. Lorch, Orbit duality for flag manifolds, Manuscripta Math. 109 (2002), $233-261$.

Che46 C. Chevalley, Theory of Lie groups (Princeton University Press, Princeton, NJ, 1946).

Fuj78 A. Fujiki, On automorphism groups of compact Kähler manifolds, Invent. Math. 44 (1978), $225-258$.

GS82 V. Guillemin and S. Sternberg, Convexity properties of the moment mapping, Invent. Math. 67 (1982), 491-513.

GS84 V. Guillemin and S. Sternberg, A normal form for the moment map, in Differential geometric methods in mathematical physics, Jerusalem, 1982, Mathematical Physics Studies, vol. 6 (Reidel, Dordrecht, 1984), 161-175.

HH96 P. Heinzner and A. Huckleberry, Kählerian potentials and convexity properties of the moment map, Invent. Math. 126 (1996), 65-84.

HS07a P. Heinzner and G. Schwarz, Cartan decomposition of the moment map, Math. Ann. 337 (2007), 197-232.

HS07b P. Heinzner and H. Stötzel, Semistable points with respect to real forms, Math. Ann. 338 (2007), $1-9$.

Hoc65 G. Hochschild, The Structure of Lie Groups (Holden-Day, San Francisco, CA, 1965).

Kir84a F. Kirwan, Cohomology of Quotients in Symplectic and algebraic geometry, Mathematical Notes, vol. 31 (Princeton University Press, Princeton, NJ, 1984).

Kir84b F. Kirwan, Convexity properties of the moment mapping. III, Invent. Math. 77 (1984), 547-552.

Kos73 B. Kostant, On convexity, the Weyl group and the Iwasawa decomposition, Ann. Sci. École Norm. Sup. (4) 6 (1973), 413-455. 


\section{STRATIFICATIONS}

Mar01 A. Marian, On the real moment map, Math. Res. Lett. 8 (2001), 779-788.

Mar84 C.-M. Marle, Le voisinage d'une orbite d'une action hamiltonienne d'un groupe de Lie, in South Rhone seminar on geometry, II, Lyon, 1983, Traveaux en Cours (Hermann, Paris, 1984), 19-35.

Mat82 T. Matsuki, Orbits on affine symmetric spaces under the action of parabolic subgroups, Hiroshima Math. J. 12 (1982), 307-320.

MUV92 I. Mirković, T. Uzawa and K. Vilonen, Matsuki correspondence for sheaves, Invent. Math. 109 (1992), 231-245.

Nee85 A. Neeman, The topology of quotient varieties, Ann. of Math. (2) 122 (1985), 419-459.

Nes84 L. Ness, A stratification of the null cone via the moment map, with an appendix by David Mumford, Amer. J. Math. 106 (1984), 1281-1329.

OS00 L. O'Shea and R. Sjamaar, Moment maps and Riemannian symmetric pairs, Math. Ann. 317 (2000), 415-457.

Sch06 P. Schützdeller, Convexity properties of moment maps of real forms acting on Kählerian manifolds, Dissertation (2006), Ruhr-Universität Bochum.

Sch89 G. Schwarz, The topology of algebraic quotients, in Topological methods in algebraic transformation groups, New Brunswick, NJ, 1988, Progress in Mathematics, vol. 80 (Birkhäuser, Boston, MA, 1989), $135-151$.

Som75 A. J. Sommese, Extension theorems for reductive group actions on compact Kähler manifolds, Math. Ann. 218 (1975), 107-116.

Wol69 J. A. Wolf, The action of a real semisimple group on a complex flag manifold. I. Orbit structure and holomorphic arc components, Bull. Amer. Math. Soc. 75 (1969), 1121-1237.

Peter Heinzner heinzner@cplx.rub.de

Fakultät für Mathematik, Ruhr-Universität Bochum, Universitätsstrasse 150, D-44780 Bochum, Germany

Gerald W. Schwarz schwarz@brandeis.edu

Department of Mathematics, Brandeis University, PO Box 549110, Waltham, MA 02454-9110, USA

Henrik Stötzel henrik.stoetzel@rub.de

Fakultät für Mathematik, Ruhr-Universität Bochum, Universitätsstrasse 150, D-44780 Bochum, Germany 